Bull. Soc. math. France

129 (2), 2001, p. 259-284

\title{
SUR CERTAINS PSEUDOGROUPES DE BIHOLOMORPHISMES LOCAUX DE $\left(\mathbb{C}^{n}, 0\right)$
}

\author{
PAR Michel Belliart
}

\begin{abstract}
RÉSUMÉ. - On montre que si $\Gamma$ est un pseudogroupe de transformations locales holomorphes de $\mathbb{C}^{n}$ en zéro contenant deux éléments "en position générale" et proches de l'identité, alors : 1) L'action de $\Gamma$ sur le fibré des jets d'ordre infini sur un petit voisinage épointé $\mathcal{B}$ de 0 est minimale (c'est-à-dire que si $z_{0}, z_{1} \in \mathcal{B}$ et si $\phi: z_{0} \rightarrow z_{1}$ est un germe de biholomorphisme alors il existe une suite $\gamma_{n} \in \Gamma$ qui converge vers $\phi$ uniformément au voisinage de $\left.z_{0}\right)$. 2) $\Gamma$ ne préserve aucune structure géométrique au voisinage de 0 (c'est une conséquence triviale du point 1). 3) Si un autre pseudogroupe holomorphe est topologiquement conjugué à $\Gamma$ alors la conjugaison est ou bien holomorphe, ou bien antiholomorphe. L'ingrédient principal de la preuve est la construction, pour tout pseudogroupe $\Gamma$, d'un faisceau $\mathfrak{g}_{\Gamma}$ d'algèbres de Lie sur $\mathbb{C}^{n}$ dans lequel $\Gamma$ est "dense" en un sens naturel. Ensuite, on prouve que si $\Gamma$ satisfait une hypothèse naturelle alors $\mathfrak{g}_{\Gamma}(U)$ contient tout champ de vecteur holomorphe sur $U$, pour tout $U$ ouvert dans $\mathcal{B}$ où $\mathcal{B}$ est le complémentaire (ouvert) de 0 dans son bassin d'attraction.
\end{abstract}

Texte reçu le 27 avril 2000, révisé le 24 juillet 2000

Michel Belliart, UMR AGAT, UFR de Mathématiques, Université Lille I, 59655 Villeneuve d'Ascq Cedex (France) • E-mail : michel.belliart@agat.univ-lille1.fr

Classification mathématique par sujets (2000). - 58H05, 58H15.

Mots clefs. - Pseudogroupes conformes, structure géométrique invariante. 
ABSTRACT (On certain pseudogroups of germs of biholomorphisms of $\left(\mathbb{C}^{n}, 0\right)$ ) Let $\Gamma$ be a pseudogroup of local holomorphic transformations of $\mathbb{C}^{n}$ fixing zero. We study the dynamics of $\Gamma$. We show that if $\Gamma$ contains two elements whose 2-jets are in "general position" and sufficiently near the identity, then: 1) $\Gamma$ acts minimally on the bundle of infinite-order jets on some pointed neighborhood $\mathcal{B}$ of 0 (that is to say: for any $z_{0}, z_{1} \in \mathcal{B}$ and any germ $\phi: z_{0} \rightarrow z_{1}$ of biholomorphism, there exists a sequence $\gamma_{n} \in \Gamma$ which converges to $\phi$ uniformly on some neighborhood of $z_{0}$ ). 2) $\Gamma$ preserves no geometric structure near 0 (this is a trivial consequence of 1 ). 3) For any holomorphic pseudogroup topologically conjugate to $\Gamma$, the germ of conjugacy at 0 is either holomorphic or antiholomorphic. The main feature of the proof is to attach to any pseudogroup $\Gamma$ a sheaf $\mathfrak{g}_{\Gamma}$ of Lie algebrae on $\mathbb{C}^{n}$ such that $\Gamma$ is "dense" in $\mathfrak{g}_{\Gamma}$ in a natural sense. Then we prove that under some natural assumption on $\Gamma$, $\mathfrak{g}_{\Gamma}(U)$ must be the sheaf of all holomorphic vector fields for any $U$ open in $\mathcal{B}$, where $\mathcal{B}$ is the (open) complementary of 0 in its basin of attraction.

\section{Introduction}

1.1. Position du problème. - Soit $\Gamma$ un pseudogroupe de biholomorphismes de $\mathbb{C}^{n}$ en $0 . \Gamma$ consiste donc en la donnée d'une collection $\left(\gamma_{i}, U_{i}\right)$ d'ouverts $U_{i} \ni 0$ et de fonctions holomorphes et injectives $\gamma_{i}: U_{i} \rightarrow \mathbb{C}^{n}$, $\gamma_{i}(0)=0$, vérifiant les deux axiomes :

- pour tous couples $\left(\gamma_{i}, U_{i}\right)$ et $\left(\gamma_{j}, U_{j}\right)$ de $\Gamma$, le couple $\left(\gamma_{i} \circ \gamma_{j}, U_{j} \cap \gamma_{j}^{-1}\left(U_{i}\right)\right)$ est encore dans $\Gamma$;

- pour tout couple $\left(\gamma_{i}, U_{i}\right)$ de $\Gamma$, le couple $\left(\gamma_{i}^{-1}, \gamma_{i}^{-1}\left(U_{i}\right)\right)$ est encore dans $\Gamma$.

On peut associer à $\Gamma$ le groupe $\Gamma_{0}$ des germes $\gamma_{0}$ en 0 de tous les éléments $\gamma$ de $\Gamma$. On dit que $\Gamma$ est une réalisation de $\Gamma_{0}$. Choisir une réalisation de $\Gamma_{0}$, c'est donc spécifier pour chaque germe $\gamma_{0}$ de $\Gamma_{0}$ un domaine $U$ sur lequel $\gamma_{0}$ admet un représentant $\gamma$ injectif, en sorte que les axiomes précédents soient vérifiés. Si $\Gamma^{\prime}$ est une autre réalisation de $\Gamma_{0}$ et si pour tout $\gamma_{0}$ le domaine du $\gamma^{\prime} \in \Gamma^{\prime}$ correspondant est contenu dans celui de $\gamma$, on dit que $\Gamma^{\prime}$ est une restriction de $\Gamma$. Par exemple, $\Gamma^{\prime}$ peut s'obtenir en posant $U_{i}^{\prime}=U_{i} \cap D$ où $D$ est un domaine fixé.

L'intérêt des notions précédentes se justifie par exemple par leur application à la théorie des feuilletages transversalement holomorphes, où les pseudogroupes d'holonomie des feuilles jouent un grand rôle. Que l'on se place ou non dans ce cadre, on peut se poser sur $\Gamma$ divers problèmes naturels, les plus immédiats nous semblant être les suivants :

- Quelles propriétés de $\Gamma$ ne dépendent que de $\Gamma_{0}$ ?

- Quelle est la dynamique de $\Gamma$ au voisinage de l'origine?

- Existe-t-il une structure géométrique ( $c f .[7])$ invariante par $\Gamma$ au voisinage de l'origine? (Ceci revient à étudier la dynamique de $\Gamma$, non plus dans $\mathbb{C}^{n}$,

TOME $129-2001-\mathrm{N}^{\mathrm{O}} 2$ 
mais dans un fibré de jets convenable, comme nous l'expliquerons plus loin).

- $\Gamma$ est-il "rigide"? ou plus précisément, quelles comparaisons peut-on faire entre les classes de conjugaison de $\Gamma$ pour divers ordres de régularité (mesurable, topologique, holomorphe. ..) ? Ceci est un premier pas vers la théorie des déformations de $\Gamma$.

- En parallèle avec la théorie des groupes de Lie, peut-on définir d'une façon ou d'une autre une "adhérence" et une algèbre de Lie pour $\Gamma$ ?

1.2. Le cas d'une variable. - Les pseudogroupes à une variable ont fait récemment l'objet de recherches intensives et sont maintenant bien compris. Leur caractère le plus marquant est la dichotomie suivante :

- Si $\Gamma_{0}$ est résoluble, alors $\Gamma$ est en fait métabélien et possède une forme normale très simple et explicite (voir [5]).

- Si $\Gamma_{0}$ n'est pas résoluble, alors :

$\triangleright$ Il existe un ouvert $\Omega$ dense dans un voisinage de 0 , ayant un nombre fini de composantes connexes $\Omega_{1}, \ldots, \Omega_{p}$, et sur chacune d'elles $\Gamma$ est minimal (c'est-à-dire à orbites toutes denses). C'est le théorème de Scherbakhov, [12].

$\triangleright$ Le complémentaire de $\Omega$ dans son adhérence est la trace sur celle-ci d'une variété analytique réelle $V_{\Gamma}$ dont le germe en 0 ne dépend en un sens que de $\Gamma_{0}$ : précisément, on a $V_{\Gamma} \subset V_{\Lambda}$ si $\Lambda$ est une restriction de $\Gamma$, et il existe une variété maximale $V_{0}$ ne dépendant que de $\Gamma_{0}$ telle que $V_{\Gamma} \subset V_{0}$. On a $V_{\Lambda}=V_{0}$ dès que $\Lambda$ est une restriction de $\Gamma$ à un domaine assez petit; enfin, si $\Gamma_{0}$ contient un couple d'éléments génériques alors $V_{\Gamma}=\{0\}$ (Nakaï, [11]).

$\triangleright$ Enfin, en se basant sur les résultats de Nakaï, I. Liousse, F. Loray et l'auteur ont montré dans [2] que sur chaque $\Omega_{i}, \Gamma$ est infiniment transitif au sens suivant : pour tous $z_{0}, z_{1} \in \Omega_{i}$ et tout germe $\phi(z)=z_{1}+a\left(z-z_{0}\right)+\cdots$ de biholomorphisme local il existe une suite $\gamma_{n} \in \Gamma$ qui converge vers $\phi$ uniformément sur un petit voisinage de $z_{0} . \Gamma$ ne préserve donc aucune structure géométrique au voisinage de $z_{0}$ en dehors de la structure complexe donnée, puisqu'une telle structure aurait un groupe d'isométries local plus petit que le groupe $\operatorname{Diff}\left(\mathbb{C}, z_{0}\right)$ des transformations locales holomorphes fixant $z_{0}$. Une autre façon d'énoncer ce résultat est de dire que l'action de $\Gamma$ est minimale dans le fibré des jets d'ordre infini sur $\Omega_{i}$.

1.3. Le cas de plusieurs variables. Énoncé des résultats. - La dichotomie précédente est propre à la dimension 1 ; en dimension $n \geq 2$, d'une part les groupes résolubles ne sont plus nécessairement métabéliens ni de dimension finie, d'autre part les groupes non-résolubles peuvent être finis en cardinal comme 
en dimension, et surtout, des groupes de dimension infinie peuvent préserver une structure géométrique ne dérivant pas de la structure complexe (feuilletage, forme de contact, forme de volume...) Par ailleurs, il n'est plus vrai que $\Gamma_{0}$ est dense dans Diff $\left(\mathbb{C}^{n}, 0\right)$ pour un choix générique (au sens de Baire) des 3 -jets de ses générateurs ${ }^{(1)}$. De fait, soit $\Lambda$ un sous-groupe de $\operatorname{Schottky~de~} \operatorname{SL}(n, \mathbb{C})$ engendré par les transformations $\lambda_{1}, \ldots, \lambda_{p}$ : si $\gamma_{1}, \ldots, \gamma_{p}$ sont des germes proches de $\lambda_{1}, \ldots, \lambda_{p}$ on montre facilement que le sous-groupe de $\operatorname{Diff}\left(\mathbb{C}^{n}, 0\right)$ engendré par les $\gamma_{i}$ est discret. L'ensemble des $p$-uples de germes engendrant un groupe discret contient donc un ouvert de $\left(\operatorname{Diff}\left(\mathbb{C}^{n}, 0\right)\right)^{p}$.

Pour autant, il reste possible de sauver quelque chose de [2]. Définissons le bassin d'attraction $\mathcal{B}$ de 0 pour $\Gamma$ comme l'ensemble des $z \in \mathbb{C}^{n}-\{0\}$ pour lesquels il existe une suite $\gamma_{n}$ d'éléments de $\Gamma$ vérifiant $\lim _{n \rightarrow+\infty} \gamma_{n}(z)=0$ (c'est donc encore l'ensemble des points dont l'orbite a l'origine dans son adhérence). Introduisons aussi, pour tout $k \in \mathbb{N}$, le groupe $G^{k}$ des $k$-jets de germes de biholomorphismes de $\mathbb{C}^{n}$ en 0 . Ainsi, $G^{k}$ est l'ensemble des transformations formelles $T$ suivantes,

$$
x_{i}^{\prime}=\sum_{p=1}^{k}\left(\sum_{j_{1}, \ldots, j_{p}} \alpha(p)_{i}^{j_{1}, \ldots, j_{p}} x_{j_{1}} \cdots x_{j_{p}}\right)
$$

où $\left(x_{1}, \ldots, x_{n}\right) \in \mathbb{C}^{n},\left(x_{1}^{\prime}, \ldots, x_{n}^{\prime}\right)=T\left(x_{1}, \ldots, x_{n}\right)$, la matrice $(\alpha(1))$ est inversible et chacun des tenseurs $(\alpha(p))$ est symétrique en ses indices supérieurs (cette dernière convention est faite pour que l'écriture de $T$ sous la forme (1) soit unique). Pour composer deux telles transformations dans $G^{k}$, on les compose d'abord comme des fonctions ordinaires puis on tronque le polynôme obtenu à l'ordre $k$. On montre que $G^{k}$ a une structure de groupe algébrique complexe. À toute fonction $f$ définie au voisinage de zéro on peut associer la suite $f_{k}$ de ses jets en ce point.

ThÉORÈme A. - Il existe dans $G^{2} \times G^{2}$ un voisinage $V$ de 1 et un ouvert de Zariski réel $Z$ tels que si $\Gamma \times \Gamma$ contient au moins un couple $\left(f_{2}, g_{2}\right) \in V \cap Z$, alors :

1) $\mathcal{B}$ est un voisinage ouvert épointé de l'origine;

2) pour tous $z_{0}, z_{1} \in \mathcal{B}$ et tout germe holomorphe et injectif $\phi$ tel que $\phi\left(z_{0}\right)=z_{1}$, il existe une suite $\gamma_{n} \in \Gamma$ qui converge vers $\phi$ uniformément sur un voisinage de $z_{0}$.

Ce théorème signifie donc que si $\Gamma$ contient deux éléments proches de l'identité et en "position générale", alors l'adhérence de $\Gamma$ contient tous les germes d'injection holomorphe entre points de $\mathcal{B}$. Par contre, il y a une obstruction à

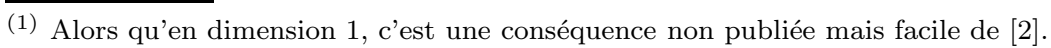

TOME $129-2001-\mathrm{N}^{\mathrm{O}} 2$ 
ce que $\Gamma$ contienne aussi tous les germes fixant 0 : c'est le déterminant de la jacobienne en 0 , qui peut être à valeurs dans un sous-groupe fermé strict de $\mathbb{C}^{*}$.

Soit $J^{k}$ le fibré des $k$-jets complexes sur $\mathcal{B}$ : c'est un $G^{k}$-fibré principal sur $\mathcal{B}$. La notion de structure géométrique, présente au moins implicitement chez Lie et Cartan, formalisée par Ehresmann et plus récemment par Gromov, est la suivante : une structure géométrique sur $\mathcal{B}$ est la donnée d'une section dans l'un des fibrés associés à l'un des $J^{k}$. Par exemple, un parallélisme est une section de $J^{1}$ car ce dernier s'identifie au fibré des repères sur $\mathcal{B}$. De ce point de vue le théorème A affirme la minimalité de $\Gamma$ sur chacun des $J^{k}$, donc admet le corollaire suivant :

THÉORÈme B. - Sous les hypothèses du théorème A, il n'existe aucune structure géométrique invariante par $\Gamma$ au voisinage de l'origine.

Pour obtenir le théorème $\mathrm{A}$, nous passons par une version infinitésimale de celui-ci, qui vaut d'être énoncée pour elle-même. Pour $U$ ouvert dans $\mathbb{C}^{n}$, soit $\mathfrak{g}(U)$ l'algèbre de Lie des champs de vecteurs holomorphes sur $U$, munie de la topologie de la convergence uniforme sur les compacts. La collection $\mathfrak{g} \operatorname{des} \mathfrak{g}(U)$ forme un faisceau sur $\mathbb{C}^{n}$. Définissons maintenant l'“adhérence" de $\Gamma$ dans $\mathfrak{g}$. Pour $X \in \mathfrak{g}(U)$ et $K \subset U$ compact, il existe un intervalle maximal $I_{K}$ de $\mathbb{R}$ sur lequel le pseudoflot $\phi_{X}^{t}$ de $X$ sur $U$ est bien défini en restriction à $K \times I_{K}$. Nous notons $\mathfrak{g}_{\Gamma}^{\prime}(U)$ l'ensemble des $X \in \mathfrak{g}(U)$ tels que pour tous $K$ et $t_{0} \in I_{K}$ tels que ci-dessus, il existe une suite $\gamma_{n}$ d'éléments de $\Gamma$ qui converge vers $\phi_{X}^{t_{0}}$ uniformément sur $K$. Un fait bien connu, que nous avons redémontré dans [2], est que pour tout $U, \mathfrak{g}_{\Gamma}^{\prime}(U)$ est une sous-algèbre de Lie réelle fermée de $\mathfrak{g}(U)$. Les $\mathfrak{g}^{\prime}$ ne forment qu'un préfaisceau, que nous complétons en un faisceau $\mathfrak{g}$ de la manière habituelle.

ThÉORÈme C. - Supposons (comme au théorème A) que $\Gamma$ contient un couple $(f, g)$ avec $\left(f_{2}, g_{2}\right) \in Z \cap V$. Alors pour tout $U$ ouvert dans $\mathcal{B}$, on a $\mathfrak{g}_{\Gamma}(U)=\mathfrak{g}(U)$.

Par contre si $U$ est un petit voisinage de l'origine, il y a une obstruction naturelle à ce que $\mathfrak{g}_{\Gamma}(U)$ contienne tous les champs de $\mathfrak{g}(U)$ nuls à l'origine : l'image de $\Gamma$ par le morphisme "déterminant" peut être un sous-groupe discret de $\mathbb{C}^{*}$. Cependant, $\mathfrak{g}_{\Gamma}(U)$ contient tout champ dont le pseudoflot n'enfreint pas cette obstruction (ce qui revient à dire : tout champ dont la partie linéaire en zéro a une trace nulle). La preuve de ce fait est une étape de la preuve du théorème $\mathrm{C}$.

Enfin, des résultats précédents découle facilement le théorème de rigidité suivant :

BULLETIN DE LA SOCIÉtÉ MATHÉMATIQUE DE FRANCE 
ThÉORÈme D. - Soient $\Gamma$ et $\Gamma^{\prime}$ deux pseudogroupes de $\left(\mathbb{C}^{n}, 0\right)$. Si $\Gamma$ vérifie les hypothèses des théorèmes $A-B-C$ et si $\Gamma^{\prime}$ lui est conjugué au voisinage de l'origine par un germe de fonction continue, alors ce germe est en fait holomorphe ou antiholomorphe.

Ce théorème de rigidité peut s'énoncer aussi en termes de feuilletages (si deux feuilletages transversalement holomorphes sont topologiquement conjugués et si le pseudogroupe d'holonomie d'une feuille de l'un vérifie nos hypothèses, alors la conjugaison est transversalement holomorphe ou anti-holomorphe). Dans cette direction, il faut citer un résultat récemment annoncé par F. Loray et J. Rebelo, qui affirme l'existence pour tout $n \geq 2$ d'un ouvert $O$ dans l'espace des feuilletages holomorphes de dimension 1 sur $\mathbb{C P}^{n}$ tel que si $\mathcal{F}$ est un feuilletage de $O$, alors il est rigide au sens d'Ilyashenko (c'est à dire que si un feuilletage holomorphe est topologiquement conjugué à $\mathcal{F}$, alors il lui est conjugué par une homographie; [8]). Ce résultat, évidemment bien plus fort que la conjugaison du théorème $\mathrm{C}$, implique diverses propriétés ergodiques des feuilletages en question; voir [10].

Pour conclure, on sait que pour $n \geq 2$, le groupe $\operatorname{Diff}\left(\mathbb{C}^{n}\right)$ des biholomorphismes globaux de $\mathbb{C}^{n}$ est de dimension infinie. Vu les résultats de densité locale obtenus ici, la question la plus naturelle est la suivante : lorsque $f$ et $g$ sont des difféomorphismes globaux, est-ce que $\Gamma$ est dense dans le stabilisateur de zéro dans Diff $\left(\mathbb{C}^{n}\right)$ ? La réponse à cette question est négative au moins pour $n=2$, comme l'a montré S. Lamy dans [9].

Remerciements. - Cet article fait suite à une question que m'a posée E. Ghys après un exposé de [2]. I. Liousse m'a convaincu de le rédiger. Je les remercie pour leur intérêt. Je remercie aussi Y. Hantout pour plusieurs discussions intéressantes.

Plan de cet article. - Le paragraphe 2 contient la définition des ouverts $Z$ et $V$; on y montre aussi que si $\Gamma$ contient un couple $\left(f_{2}, g_{2}\right) \in Z \cap V$ alors sa projection sur $G^{2}$ y est Zariski-dense. Dans le paragraphe 3, on étudie $\mathfrak{g}_{\Gamma}$ en détail. Dans le paragraphe 4 on tire des résultats précédemment démontrés la preuve des théorèmes $\mathrm{A}$ et $\mathrm{C}$; le théorème $\mathrm{D}$ est démontré au paragraphe 5 . Le théorème $\mathrm{B}$ est quant à lui une simple traduction d'une partie du théorème $\mathrm{A}$ en termes de structures géométriques et on ne le prouvera donc pas.

\section{Définition de $Z$ et $V$}

Comme nous nous adressons plutôt à un public de dynamiciens, nous allons donner des preuves détaillées de faits qui paraîtront sans doute triviaux aux spécialistes de géométrie algébrique. Rien de ce qui suit n'est très nouveau.

TOME $129-2001-\mathrm{N}^{\mathrm{O}} 2$ 
2.1. Généralités sur les groupes algébriques. - Dans ce paragraphe, tous les espaces topologiques considérés sont des variétés algébriques complexes lisses ; considérons la topologie de Zariski $\mathcal{T}_{1}$ d'un tel espace : il est connu que celle-ci est accessible ${ }^{(2)}$ mais non-séparée, strictement moins fine que la topologie de variété différentielle $\mathcal{T}_{2}$ que possède également la variété considérée. Ensuite, considérons la topologie de Zariski réelle $\mathcal{T}_{3}$ : on peut vérifier que celle-ci est plus fine que $\mathcal{T}_{2}$ et moins fine que $\mathcal{T}_{1}$. Nous serons amenés à utiliser tantôt l'une, tantôt l'autre de ces trois topologies, ce qui nous amènera à prendre de nombreuses précautions oratoires.

Rappelons ensuite qu'un espace topologique $X$ est quasi-compact s'il vérifie l'axiome de Borel-Lebesgue : de tout recouvrement ouvert de $X$ on peut extraire un recouvrement fini. Un espace compact est un espace quasi-compact séparé ; un espace quasi-compact pour la topologie $\mathcal{T}$ est encore quasi-compact pour toute topologie $\mathcal{T}^{\prime}$ strictement moins fine que $\mathcal{T}$. Enfin, on montre aisément que si $X$ est un espace topologique quasi-compact et $Y$ un espace quelconque, la projection canonique de $X \times Y$ sur $Y$ est fermée. Une conséquence directe de ce qui précède est que les variétés algébriques projectives complexes sont quasi-compactes à la fois pour leurs trois topologies (de Zariski complexe, de Zariski réelle, de sous-espace de la variété différentielle $\left.\mathbb{P} \mathbb{C}^{n}\right)$. Ceci s'applique en particulier aux grassmaniennes de $k$-plans de $\mathbb{C}^{n}$.

Un groupe algébrique est une variété algébrique munie d'une structure de groupe topologique compatible avec sa topologie de Zariski. Un sous-groupe algébrique du groupe algébrique $G$ est bien sûr un sous-groupe de $G$ qui en est aussi une sous-variété. Un groupe algébrique complexe a une structure naturelle de groupe de Lie complexe, et tout sous-groupe algébrique de $G$, puisque fermé dans $G$, est également un sous-groupe de Lie de $G$.

EXEMPLES 2.1.1. - Le groupe multiplicatif $\left(\mathbb{C}^{*}\right)^{n}$ est algébrique, car il s'identifie à la variété des points $\left(x_{1}, \ldots, x_{n}, y\right)$ de $\mathbb{C}^{n+1}$ qui vérifient $x_{1} \cdots x_{n} y=1$ munie d'une loi de groupe polynomiale. De même, $\operatorname{GL}(n, \mathbb{C})$ est algébrique lorsqu'on l'identifie à la variété des $\left(n^{2}+1\right)$-uples $\left(\alpha_{i}^{j}, a\right)$ tels que $a \operatorname{det}\left(\alpha_{i}^{j}\right)=1$. $\mathrm{Vu}$ comme groupe des matrices diagonales, $\left(\mathbb{C}^{*}\right)^{n}$ est un sous-groupe algébrique de $\operatorname{GL}(n, \mathbb{C})$. De même, $\operatorname{SL}(n, \mathbb{C})$ est un sous-groupe algébrique de $\operatorname{GL}(n, \mathbb{C})$.

Si $\Gamma$ est un sous-groupe du groupe algébrique complexe $G$, on appellera adhérence son adhérence dans $G$ pour la topologie de groupe de Lie, clôture de Zariski réelle son adhérence en topologie de Zariski réelle et clôture de Zariski son adhérence pour la topologie de Zariski complexe. Ce sont trois sous-groupes de $G$; l'adhérence de $\Gamma$ est un sous-groupe de Lie de $G$ et sa clôture de Zariski en est un sous-groupe algébrique. La clôture de Zariski (réelle ou complexe)

$\overline{(2)}$ Un espace $X$ est accessible si pour tout couple de points $x, y$ de $X$, l'ensemble $X-\{x\}$ est un voisinage de $y$, ce qui revient à dire que le singleton $\{x\}$ est fermé. 
de $\Gamma$ contient son adhérence mais peut être plus grande, comme le révèle le fait suivant (voir [3], 8.8, p. 116).

Proposition 2.1.2. — Dans $\left(\mathbb{C}^{*}\right)^{n}$, le groupe monogène engendré par $\left(x_{1}, \ldots, x_{n}\right)$ (qui n'est jamais dense en topologie ordinaire) est Zariski-dense si et seulement si les nombres $x_{1}, \ldots, x_{n}$ sont rationnellement indépendants.

D'autre part, en considérant le cas de $\operatorname{GL}(n, \mathbb{R}) \subset \mathrm{GL}(n, \mathbb{C})$ on constate que la clôture de Zariski réelle de $\Gamma$ peut être strictement plus petite que sa clôture de Zariski.

Si $\Gamma$ est un sous-groupe Zariski-fermé du groupe algébrique $G$, alors $\Gamma$ est un sous-groupe de Lie fermé du groupe de Lie sous-jacent à $G$ et on peut montrer que dans l'une ou l'autre topologie, $\Gamma$ n'a qu'un nombre fini de composantes connexes (voir [3], p. 46). En particulier, l'algèbre de Lie de la clôture de Zariski d'un sous-groupe infini de $G$ n'est pas nulle.

2.2. Le groupe (algébrique) $\mathbf{G L}(\boldsymbol{n}, \mathbb{C})$. - Dans toute la suite, nous notons $\left(X_{i}^{j}\right)$ la base usuelle de l'algèbre de Lie $\mathfrak{g l}(n, \mathbb{C})$ de $\operatorname{GL}(n, \mathbb{C})$. Comme on sait, cette algèbre s'identifie à celle des matrices $n \times n$ à coefficients complexes, le crochet étant défini par la formule $[A, B]=A B-B A$. Alors, $X_{i}^{j}$ est la matrice qui a un ' 1 ' en ligne $i$ et colonne $j$, et les autres termes nuls. Pour l'action usuelle de $\operatorname{GL}(n, \mathbb{C})$ sur $\mathbb{C}^{n}$, le champ fondamental associé à $X_{i}^{j}$ est $x_{i} \partial / \partial x_{j}$. On a la relation $\left[X_{i}^{j}, X_{k}^{\ell}\right]=\delta_{j}^{k} X_{i}^{\ell}-\delta_{i}^{\ell} X_{k}^{j}$. L'algèbre de Lie $\mathfrak{s l}(n, \mathbb{C})$ de $\operatorname{SL}(n, \mathbb{C})$ est un idéal de codimension un de $\mathfrak{g l}(n, \mathbb{C})$ et la droite $\mathfrak{c}$ des matrices d'homothétie en est un supplémentaire; $\mathfrak{c}$ est le centre de $\mathfrak{g l}(n, \mathbb{C})$ et on a $\mathfrak{g l}(n, \mathbb{C})=\mathfrak{c} \oplus \mathfrak{s l}(n, \mathbb{C})$. Enfin, on sait que l'algèbre de Lie réelle $\mathfrak{s l}(n, \mathbb{C})$ est simple, ce qui implique le fait suivant :

Lemme 2.2.1. - Soit $\Gamma$ un sous-groupe Zariski-dense de $\operatorname{GL}(n, \mathbb{C})$. Alors, pour tout $X \in \mathfrak{s l}(n, \mathbb{C})-\{0\}$, la $\Gamma$-orbite de $X$ pour l'action adjointe contient une base de l'espace vectoriel sous-jacent à $\mathfrak{s l}(n, \mathbb{C})$. De même, si $\Gamma$ est dense pour la topologie de Zariski réelle, alors la $\Gamma$-orbite de $X$ contient une base de l'espace vectoriel réel $\mathfrak{s l}(n, \mathbb{C})$.

Preuve. - Que le corps de base choisi soit $\mathbb{R}$ ou $\mathbb{C}$, l'hypothèse est que $\Gamma$ est dense dans $\operatorname{GL}(n, \mathbb{C})$. Or, l'orbite de $X$ engendre un sous-espace vectoriel $E$ non-nul de $\mathfrak{s l}(n, \mathbb{C})$ invariant par $\Gamma$, donc par sa clôture de Zariski. On doit donc avoir $E=\mathfrak{s l}(n, \mathbb{C})$.

Proposition 2.2.2. - Soit $\mathbb{K}=\mathbb{R}$ ou $\mathbb{C}$. L'ensemble $Z_{1}(\mathbb{K})$ des couples $(f, g) \in \operatorname{GL}(n, \mathbb{C}) \times \operatorname{GL}(n, \mathbb{C})$ qui ne fixent aucun $\mathbb{K}$-sous-espace vectoriel propre de $\mathfrak{s l}(n, \mathbb{C})$ pour la représentation adjointe est un ouvert de Zariski (non vide), et si $(f, g)$ est dans $Z_{1}(\mathbb{K})$ alors le sous-groupe $\Gamma$ de $\operatorname{GL}(n, \mathbb{C})$ engendré par $f$ et $g$ ou bien est fini, ou bien contient $\mathrm{SL}(n, \mathbb{C})$ dans sa $\mathbb{K}$-clôture de Zariski.

TOME $129-2001-\mathrm{N}^{\mathrm{O}} 2$ 
Preuve. - Dire que $f$ et $g$ laissent invariant le même $\mathbb{K}$-sous-espace $\mathfrak{h}$ de $\mathfrak{s l}(n, \mathbb{C})$ revient à dire que $f$ et $g$ fixent le point $x$ correspondant à $\mathfrak{h}$ dans la grassmanienne $\mathbb{G}^{\operatorname{dim}(\mathfrak{h})}(\mathfrak{s l}(n, \mathbb{C}))$ des $\mathbb{K}$-sous-espaces de dimension $\operatorname{dim}(\mathfrak{h})$. L'ensemble $F_{\operatorname{dim}(\mathfrak{h})}$ des triplets $(f, g, x)$ tels que $f x=g x=x$ est fermé dans $G \times G \times \mathbb{G}^{\operatorname{dim}(\mathfrak{h})}(\mathfrak{s l}(n, \mathbb{C}))$ et $\mathbb{G}^{\operatorname{dim}(\mathfrak{h})}(\mathfrak{s l}(n, \mathbb{C}))$ est quasi-compacte donc chaque $F_{\operatorname{dim}(\mathfrak{h})}$ se projette sur un fermé de $G \times G$. Le complémentaire de leur réunion est donc ouvert, or c'est $Z_{1}(\mathbb{K})$. Montrons ensuite que $Z_{1}(\mathbb{C})$ n'est pas vide. Soit $f$ une matrice diagonale dont les termes diagonaux sont rationnellement indépendants. Alors la clôture de Zariski du sous-groupe engendré par $f$ est le tore $\mathbb{T}_{f}$ des matrices diagonales. Soit $M$ la matrice de $\mathfrak{g l}(n, \mathbb{C})$ dont tous les termes sont égaux à 1 : on peut montrer que $M$ est diagonalisable, donc il existe un tore $\mathbb{T}_{g}$ conjugué à $\mathbb{T}_{f}$ dont toutes les matrices commutent à $M$. Ce tore contient un élément $g$ aux valeurs propres rationnellement indépendantes. Soient $\Gamma$ le groupe engendré par $f$ et $g, G$ sa clôture de Zariski, $\mathfrak{g}$ l'algèbre de Lie de $G$. Comme la représentation adjointe de $\operatorname{GL}(n, \mathbb{C})$ dans $\mathfrak{s l}(n, \mathbb{C})$ est irréductible et qu'une sous-algèbre à la fois $f$-et $g$-invariante est $\Gamma$ - donc $G$ invariante, il suffit de montrer que $G=\operatorname{GL}(n, \mathbb{C})$. Puisque $\mathbb{T}_{f}$ est dans $G, \mathfrak{g}$ contient l'algèbre de ce tore, qui a pour base les champs $X_{i}^{i}$. Comme $\mathbb{T}_{g}$ est dans $G$ et commute à $M, \mathfrak{g}$ contient aussi $M$. On calcule que pour $i \neq j$,

$$
\begin{gathered}
{\left[X_{i}^{i},\left[X_{j}^{j}, M\right]\right]=\left[X_{i}^{i}, \sum_{k=1}^{n}\left(X_{j}^{k}-X_{k}^{j}\right)\right]=-X_{j}^{i}-X_{i}^{j},} \\
{\left[X_{i}^{i},\left[X_{i}^{i},\left[X_{j}^{j}, M\right]\right]\right]=X_{j}^{i}-X_{i}^{j}}
\end{gathered}
$$

donc $\mathfrak{g}$ contient $X_{j}^{i}$ et $X_{i}^{j}$ pour tous $i$ et $j$ distincts. On en déduit que $\mathfrak{g}=$ $\mathfrak{g l}(n, \mathbb{C})$, ce qui achève la preuve que $(f, g) \in Z_{1}(\mathbb{C})$. Déduisons-en que $Z_{1}(\mathbb{R})$ n'est pas vide non plus. Si $(f, g)$ est dans $Z_{1}(\mathbb{C})$ mais pas dans $Z_{1}(\mathbb{R})$, il existe un sous-espace réel propre $E$ de $\mathfrak{s l}(n, \mathbb{C})$ qui est $(f, g)$-invariant. De ce fait les sous-espaces complexes $E \cap i E$ et $E+i E$ sont eux aussi invariants, mais puisque $(f, g) \in Z_{1}(\mathbb{C})$ ceci implique que $E \cap i E=\{0\}$ et $E+i E=\mathfrak{s l}(n, \mathbb{C})$; soient alors $f_{0}$ et $g_{0}$ les restrictions de $f$ et $g$ à $E$ : il existe un isomorphisme entre $\mathfrak{s l}(n, \mathbb{C})$ et $E \otimes_{\mathbb{R}} \mathbb{C}$ par lequel $f$ et $g$ s'identifient à $f_{0} \otimes 1$ et $g_{0} \otimes 1$; de ceci découle que l'ensemble des valeurs propres de $f$ (ou de $g$ ) est stable par conjugaison complexe. Mais les matrices de $\operatorname{GL}(n, \mathbb{C})$ n'ayant pas cette propriété forment clairement un ouvert de Zariski réel non vide $Z$, donc $Z_{1}(\mathbb{R})$ qui contient par construction $Z_{1}(\mathbb{C}) \cap Z \times Z$ n'est pas vide. Enfin, soit $(f, g) \in$ $Z_{1}(\mathbb{K})$ et supposons que le sous-groupe $\Gamma$ engendré par $f$ et $g$ dans $\operatorname{GL}(n, \mathbb{C})$ est infini : alors sa clôture de Zariski $G$ a une algèbre de Lie $\mathfrak{g}$ non triviale, et puisque $f, g$ ne fixent aucune sous-algèbre propre de $\mathfrak{s l}(n, \mathbb{C}), \mathfrak{g}$ n'est pas non plus incluse dans $\mathfrak{c}$ (sinon, $f$ et $g$ fixeraient au contraire tout sous-espace vectoriel de $\mathfrak{s l}(n, \mathbb{C})$ puisque $\mathfrak{c}$ est central dans $\mathfrak{g l}(n, \mathbb{C}))$. Donc la projection de $\mathfrak{g} \operatorname{sur} \mathfrak{s l}(n, \mathbb{C})$ n'est pas nulle, et comme elle est $f$ - et $g$-invariante ce doit être $\mathfrak{s l}(n, \mathbb{C})$. 
2.3. Le groupe (algébrique) $\boldsymbol{G}^{\mathbf{2}}$. - Selon la formule (1), $G^{2}$ est l'ensemble des transformations formelles

$$
x_{i}^{\prime}=\sum_{j} \alpha_{i}^{j} x_{j}+\sum_{j, k} \beta_{i}^{j k} x_{j} x_{k} \quad\left(\beta_{i}^{j k}=\beta_{i}^{k j}, \operatorname{det}\left(\alpha_{i}^{j}\right) \neq 0\right) .
$$

L'application "déterminant" se prolonge de $\operatorname{GL}(n, \mathbb{C})$ à $G^{2}$, et en parallèle à la notation $\operatorname{SL}(n, \mathbb{C})$ on notera $\mathrm{SG}^{2}$ son noyau dans ce groupe. L'action naturelle de $\operatorname{GL}(n, \mathbb{C})$ sur $\mathbb{C}^{n}$ induit une représentation algébrique $\rho$ de $\operatorname{GL}(n, \mathbb{C})$ dans l'espace vectoriel $E$ des tenseurs symétriques $\beta_{i}^{j k}$ et $G^{2}$ est le produit semidirect de $E$ par $\mathrm{GL}(n, \mathbb{C})$ correspondant à $\rho$. C'est donc un groupe algébrique. Notons que l'image par $\rho$ d'une homothétie de $\operatorname{GL}(n, \mathbb{C})$ est une homothétie de même rapport dans $E$; de ce fait tout sous-espace vectoriel réel $F$ de $E$ invariant par $\rho$ est en fait un sous-espace complexe. Enfin, de même que $G^{2}$ est extension de $E$ par $\operatorname{GL}(n, \mathbb{C})$, l'algèbre $\mathfrak{g}^{2}$ de $G^{2}$ est extension de $\mathfrak{g l}(n, \mathbb{C})$ par l'algèbre $\mathfrak{e}$ de $E$. Nous notons $X_{i}^{j k}$ la base de $\mathfrak{e}$ telle que $e_{i}^{j k}$ est l'exponentielle de $X_{i}^{j k}$. Bien sûr, $E$ étant abélien, on pourrait l'identifier à $\mathfrak{e}$ et identifier dans le même élan les $X_{i}^{j k}$ aux $e_{i}^{j k}$, mais il nous semble que l'emploi de cette notation double facilite quelque peu la rédaction de la proposition 2.3.2. Nous notons $\mathfrak{s g}^{2}$ l'algèbre de $\mathrm{SG}^{2}$.

Lemme 2.3.1. - L'ensemble $Z_{2}$ des e $\in E$ dont l'orbite pour $\rho$ n'est pas contenue dans un sous-espace vectoriel strict de $E$ est un ouvert de Zariski non vide de $E$. Le sous-groupe de $E$ engendré par l'orbite d'un tel $e \in Z_{2}$ est égal à $E$.

Preuve. - Pour $k \in\{1,2, \ldots, \operatorname{dim}(E)-1\}$, soient $\mathbb{G}^{k}(E)$ la grassmanienne des $k$-plans complexes de $E$ et $F_{k}$ l'ensemble des couples $(x, y)$ de $E \times \mathbb{G}^{k}(E)$ tels que : $x$ appartient au sous-espace vectoriel de $E$ dirigé par $y$, et $y$ est $\mathrm{GL}(n, \mathbb{C})$ invariant. Alors, $F_{k}$ est fermé; donc sa projection sur $E$ l'est aussi, puisque $\mathbb{G}^{k}(E)$ est quasi-compacte. Le complémentaire de la réunion des $F_{k}$ est donc ouvert, et c'est $Z_{2}$. Montrons ensuite que $Z_{2}$ n'est pas vide. Soit $e_{i}^{j k}$ la base canonique de $E$, formée des transformations

$$
x_{i}^{\prime}=x_{i}+\sum_{k, l} \delta_{i}^{k} \delta_{j}^{\ell} x_{k} x_{\ell} .
$$

Soit $F$ le sous-espace vectoriel de $E$ engendré par la $\rho(\operatorname{GL}(n, \mathbb{C}))$-orbite de $e_{1}^{11}$. Nous montrons que $E=F$, ce qui revient bien à dire que $e_{1}^{11} \in Z_{2}$. On constate que sous l'action du groupe à un paramètre engendré par $X_{1}^{2}$, l'orbite de $e_{1}^{11}$ est la famille $\left\{e_{1}^{11}+2 t e_{1}^{12}+t^{2} e_{1}^{22}\right\}$ donc $e_{1}^{12} \in F$ et $e_{1}^{22} \in F$; en outre si $n \geq 3$, l'orbite de $e_{1}^{12}$ pour $X_{1}^{3}$ est $e_{1}^{12}+t e_{1}^{23}$ donc $e_{1}^{23} \in F$.

En faisant agir le groupe des permutations de coordonnées sur $F$, on voit donc que $F$ contient :

- l'orbite $\left\{e_{i}^{i i}\right\}$ de $e_{1}^{11}$; 
- l'orbite $\left\{e_{i}^{j j}\right\}(i \neq j)$ de $e_{2}^{11}$;

- l'orbite $\left\{e_{i}^{i j}\right\}(i \neq j)$ de $e_{1}^{12}$;

- le cas échéant, l'orbite $\left\{e_{i}^{j k}\right\}(i \neq j \neq k \neq i)$ de $e_{1}^{23}$.

Donc $F$ contient la base $\left(e_{i}^{j k}\right)$ de $E$. Ceci prouve que $e_{1}^{11} \in Z_{2}$, comme annoncé plus haut. Enfin, soit $e \in Z_{2}$ : le sous-groupe de $E$ engendré par la $\rho$-orbite de $e$ est invariant par les homothéties de $\operatorname{GL}(n, \mathbb{C})$ donc c'est un sous-espace vectoriel, et par définition il contient une base de $E$.

Proposition 2.3.2. - Soit $\Gamma$ un sous-groupe $\mathbb{R}$-Zariski-dense de $G^{2}$. Alors, pour tout $X \in \mathfrak{s l}(n, \mathbb{C})$ la $\Gamma$-orbite de $X$ dans $\mathfrak{s g}^{2}$ pour l'action adjointe contient une base de l'espace vectoriel réel sous-jacent à $\mathfrak{s g}^{2}$.

Preuve. - Par le lemme 2.2.1, l'adhérence de cette orbite contient une base de $\mathfrak{s l}(n, \mathbb{C})$. De plus elle contient l'image de $X_{1}^{1}-X_{2}^{2} \operatorname{par} \operatorname{Ad}\left(e_{1}^{11}\right)$ et un rapide calcul montre que cette image est $X_{1}^{1}-X_{2}^{2}+X_{1}^{11}$. Or selon le lemme 2.3.1, la $\Gamma$-orbite de $X_{1}^{11}$ contient une base de $\mathfrak{e}$ puisque celle de $e_{1}^{11}$ en contient une de $E$. On a donc le résultat voulu.

2.4. Définition de $Z$ et $V$; adhérence de $\Gamma$ dans $G^{2}$. - Soit $\mathbb{A}$ l'espace affine sous-jacent à $E$, qu'on voit comme le quotient $G^{2} / \mathrm{GL}(n, \mathbb{C})$, de sorte que $G^{2}$ agit sur $\mathbb{A}$ par transformations affines et $E$ par translations. À tout $a \in \mathbb{A}$ on peut associer son stabilisateur $G(a)$ dans $G^{2}$, qui est une section de la projection canonique $\pi$ de $G^{2}$ sur GL $(n, \mathbb{C})$. D'autre part on peut vérifier que si la matrice $\left(\alpha_{i}^{j}\right)$ a les valeurs propres $\lambda_{1}, \ldots, \lambda_{n}$ alors $\rho\left(\alpha_{i}^{j}\right)$ a les valeurs propres $\lambda_{1}, \ldots, \lambda_{n}$ ( $n$ fois chacune) et $\lambda_{i}^{-1} \lambda_{j} \lambda_{k}$ (une fois chacune pour $i \notin\{j, k\}$ ). De ceci on déduit que dans $\mathrm{GL}(n, \mathbb{C})$, l'ouvert $Z_{3}$ des $\left(\alpha_{i}^{j}\right)$ telles que 1 ne soit pas valeur propre de $\rho\left(\alpha_{i}^{j}\right)$ est non-vide. Or, un élément $\alpha$ de $G^{2}$ tel que $\pi(\alpha) \in Z_{3}$ n'a qu'un point fixe dans $\mathbb{A}$ : donc, on peut définir une application $P(\alpha)$ de $\pi^{-1}\left(Z_{3}\right)$ dans $\mathbb{A}$ qui à $\alpha$ associe son unique point fixe.

DÉfinition 2.4.1. - Nous appelons $Z$ l'ouvert de Zariski réel de $G^{2} \times G^{2}$ formé des couples $\left(f_{2}, g_{2}\right)$ qui vérifient les cinq conditions suivantes (la quatrième d'entre elles sert à assurer que la cinquième a un sens) :

1) $\left|\operatorname{det}\left(f_{2}\right)\right| \neq\left|\operatorname{det}\left(g_{2}\right)\right|$;

2) l'une des matrices $\pi\left(f_{2}\right)$ ou $\pi\left(g_{2}\right)$ a deux valeurs propres de module différent ;

3) $\left(\pi\left(f_{2}\right), \pi\left(g_{2}\right)\right) \in Z_{1}(\mathbb{R})$;

4) $\pi\left(f_{2}\right) \in Z_{3}$ et $\pi\left(g_{2}\right) \in Z_{3}$;

5) $P\left(g_{2}\right)-P\left(f_{2}\right) \in Z_{2}$.

Enfin, soient $f_{2}, g_{2} \in G^{2}$ et soit $\Gamma$ le sous-groupe de $G^{2}$ engendré par $f_{2}$ et $g_{2}$. On peut appliquer un théorème connu de Zassenhaus (voir [6] pour l'énoncé 
complet, une preuve simple et une généralisation) : il existe un voisinage $V$ de 1 dans $G^{2} \times G^{2}$ tel que si $\left(f_{2}, g_{2}\right) \in V$ alors $\Gamma$ est soit nilpotent, soit non-discret.

Proposition 2.4.2. - Si $\left(f_{2}, g_{2}\right) \in Z \cap V$ alors l'adhérence de $\Gamma$ dans $G^{2}$ contient à la fois $\mathrm{SG}^{2}$ et une homothétie dont le rapport n'est pas de module 1 .

Preuve. - Soient $G$ l'adhérence de $\Gamma$ et $H$ sa clôture de Zariski réelle. La condition 2) de 2.4.1 fait que $H$ est infini et non-central dans $\operatorname{GL}(n, \mathbb{C})$; alors, la condition 3) implique que $\pi(H) \supset \mathrm{SL}(n, \mathbb{C})$ en vertu de la proposition 2.2.2. D'autre part, la condition 1) implique que $\operatorname{det}(\pi(H))$ est infini dans $\mathbb{C}^{*}$ donc égal à ce groupe. Par conséquent $\pi(H)=\mathrm{GL}(n, \mathbb{C})$. Ensuite, soit $K=G \cap E$ : $K$ est $\rho(\pi(H))$-invariant; de la condition 4$)$ et du lemme 2.3 .1 résulte donc que $K=E$. Soit $\mathfrak{g}$ l'algèbre de Lie de la projection de $G \operatorname{sur} \operatorname{SL}(n, \mathbb{C}): \mathfrak{g}$ est invariante par $\pi(H)$, et comme $\Gamma$ n'est pas nilpotent (sinon $H$ le serait), le lemme de Zassenhaus implique que $\mathfrak{g}$ n'est pas nulle. On a donc $\mathfrak{g}=\mathfrak{s l}(n, \mathbb{C})$ en vertu du lemme 2.2.1.

\section{3. Étude du faisceau $\mathfrak{g}_{\Gamma}$}

3.1. Préliminaires sur g. - Pour $U$ ouvert dans $\mathbb{C}^{n}$ et $k \geq 0$, soit $\mathfrak{g}_{k}(U)$ l'espace vectoriel des restrictions à $U$ de champs de vecteurs homogènes de degré $k$ sur $\mathbb{C}^{n}$. Une base naturelle pour $\mathfrak{g}_{k}(U)$ est la collection des (restrictions à $U$ des) champs de la forme

$$
X_{i}^{j_{1} \ldots j_{n}}=x_{1}^{j_{1}} \cdots x_{n}^{j_{n}} \frac{\partial}{\partial x_{i}} \quad\left(1 \leq i \leq n, \sum_{\ell=1}^{n} j_{\ell}=k+1\right)
$$

Ainsi, $\mathfrak{g}_{0}(U)$ s'identifie à $\mathfrak{g l}(n, \mathbb{C})$ vue comme algèbre des champs fondamentaux de l'action usuelle de $\operatorname{GL}(n, \mathbb{C})$. Par contre, $\mathfrak{g}_{k}(U)$ n'est pas une algèbre de Lie pour $k \geq 1$. Bien au contraire :

Proposition 3.1.1. - Soient $U$ un ouvert de $\mathbb{C}^{n}, \mathfrak{h}(U)$ une sous-algèbre de Lie de $\mathfrak{g}(U)$ et supposons que $\mathfrak{g}_{p}(U) \subset \mathfrak{h}(U)$ pour un certain $p \geq 1$ : alors, on a aussi $\mathfrak{g}_{k p}(U) \subset \mathfrak{h}(U)$ pour tout $k \geq 2$.

Preuve. - Vu ce qu'il faut montrer, on peut considérer que $\mathfrak{h}(U)$ est la plus petite sous-algèbre de $\mathfrak{g}(U)$ contenant $\mathfrak{g}_{p}(U)$. Comme $\mathfrak{g}_{p}(U)$ est invariante par l'action adjointe de $\mathfrak{g}_{0}(U)$, l'identité de Jacobi implique alors que $\mathfrak{h}(U)$ est elleaussi invariante. De même, elle est invariante par le groupe des permutations de coordonnées car c'est le cas de $\mathfrak{g}_{p}(U)$. Ceci étant, les deux formules

$$
\begin{gathered}
{\left[x_{i}^{p+1} \frac{\partial}{\partial x_{j}}, x_{i}^{p} x_{j} \frac{\partial}{\partial x_{i}}+\frac{p+1}{2} x_{i}^{p-1} x_{j}^{2} \frac{\partial}{\partial x_{j}}\right]=x_{i}^{2 p+1} \frac{\partial}{\partial x_{i}} \quad(i \neq j),} \\
{\left[x_{i}^{p+1} \frac{\partial}{\partial x_{i}}, x_{i}^{k p+1} \frac{\partial}{\partial x_{i}}\right]=(k-1) p x_{i}^{k p+p+1} \frac{\partial}{\partial x_{i}}}
\end{gathered}
$$

TOME $129-2001-\mathrm{N}^{\mathrm{O}} 2$ 
impliquent que $\mathfrak{h}(U)$ contient tous les champs de la forme $x_{i}^{k p+1} \partial / \partial x_{i}$ pour $k \geq 1$. Soit maintenant $\left(i_{1}, \ldots, i_{n}\right)$ un $n$-uple et $i=i_{1}+\cdots+i_{n}=k p+1$. Si l'on itère $i$ fois l'opération de crochet $\left[x_{1} \partial / \partial x_{n},.\right]$ à partir de $x_{n}^{i} \partial / \partial x_{n}$, on obtient

$$
\frac{n !}{\left(n-i_{1}\right) !} x_{1}^{i_{1}} x_{n}^{n-i_{1}} \frac{\partial}{\partial x_{n}} .
$$

$\mathrm{Si}$ on continue en appliquant successivement par induction sur $k<n$ le crochet itéré $i_{k}$ fois avec $x_{k} \partial / \partial x_{n}$, on obtient en fin de compte le champ $\left(n ! / i_{n} !\right) x_{1}^{i_{1}} \cdots x_{n}^{i_{n}} \partial / \partial x_{n}$ qui est donc dans $\mathfrak{h}(U)$. En faisant ensuite agir le groupe des permutations de coordonnées, on voit que $\mathfrak{h}(U)$ contient tous les champs de la forme $\left(2_{k p}\right)$.

Le résultat précédent a pour défaut de ne pas être intrinsèque : en effet, la définition de $\mathfrak{g}_{\mathfrak{i}}$ dépend du choix de coordonnées locales en zéro (elle n'est invariante que par transformations linéaires). Par contre, en tronquant le crochet de Lie à l'ordre homogène $i+1$, on fait de $\mathfrak{g}^{i}=\bigoplus_{j<i} \mathfrak{g}_{j}$ une algèbre de Lie qui s'identifie de façon naturelle à celle de $G^{i+1}$. Si $U$ est un voisinage ouvert de 0 , l'algèbre $\mathfrak{g}(U, 0)$ des champs de $\mathfrak{g}(U)$ qui s'annulent en 0 se projette sur $\mathfrak{g}^{i}$. Comme on sait, le noyau de cette projection ne dépend pas des coordonnées locales utilisées. Le résultat précédent implique alors :

Proposition 3.1.2. - Soit $U$ un voisinage ouvert de 0 . Si la sous-algèbre $\mathfrak{h}(U, 0)$ de $\mathfrak{g}(U, 0)$ se surjecte sur $\mathfrak{g}^{1}$ alors sa restriction à toute boule $\mathbb{B}$ centrée en 0 et suffisamment petite est dense dans $\mathfrak{g}(\mathbb{B}, 0)$.

Preuve. - Par hypothèse, $\mathfrak{h}(U, 0)$ contient un champ de la forme

$$
X=\sum_{i=1}^{n} X_{i}^{i}+\cdots
$$

où les pointillés désignent les termes non-linéaires. Un tel champ se linéarise au voisinage de l'origine. On peut donc après un changement de coordonnées convenable supposer que

- $U$ est la boule unité $\mathbb{B}$ de $\mathbb{C}^{n}$;

- $X=\sum_{i=1}^{n} X_{i}^{i}$ sur $\mathbb{B}$.

Rappelons ensuite la formule de Stirling :

$$
k !=k^{k+\frac{1}{2}} \mathrm{e}^{-k} \sqrt{2 \pi}(1+\varepsilon(k)) .
$$

Notons que pour $k$ fixé et $p$ variable, le coefficient binomial $C_{k}^{p}$ atteint son maximum lorsque $2 p \leq k \leq 2 p+1$. De (3) découle donc que

$$
\sup _{p \in \mathbb{N}} C_{2 k}^{p} \leq \frac{(2 k)^{2 k+\frac{1}{2}} \mathrm{e}^{-2 k} \sqrt{2 \pi}}{\left(k^{k+\frac{1}{2}} \mathrm{e}^{-k} \sqrt{2 \pi}\right)^{2}}(1+\varepsilon(k)) \leq \frac{4^{k}}{\sqrt{k \pi}}(1+\varepsilon(k))=o\left(4^{k}\right) .
$$

BULletin DE LA SOCiÉtÉ MATHÉmATiQUe DE FRANCE 
Et de même,

$$
\sup _{p \in \mathbb{N}} C_{2 k+1}^{p} \leq \sup _{p \in \mathbb{N}} C_{2 k+2}^{p}=o\left(4^{k}\right) .
$$

Donc $\sup _{p \in \mathbb{N}} C_{k}^{p}=o\left(2^{k}\right)$.

À présent, soit $Y_{0,0} \in \mathfrak{g}_{0}$ et montrons que $Y_{0,0} \in \mathfrak{h}(\varepsilon \mathbb{B}, 0)$ pour $\varepsilon$ assez petit. Pour cela, nous choisissons dans $\mathfrak{h}$ un élément $Y_{0}$ de la forme $Y_{0}=$ $Y_{0,0}+Y_{0,1}+\cdots$ où $Y_{0, i}$ est homogène de degré $i$, ce que les hypothèses de la proposition nous autorisent à faire. Ensuite, nous posons par récurrence

$$
Y_{k}=\sum_{i \in \mathbb{N}} Y_{k, i}=Y_{k-1}-\frac{1}{k}\left[X, Y_{k-1}\right] \quad(k>0) .
$$

Un calcul direct donne alors

$$
Y_{k}=Y_{0,0}+\sum_{\ell>k}(-1)^{k} C_{\ell-1}^{k} Y_{0, \ell}
$$

Ceci montre que la suite $Y_{k}$ converge formellement vers $Y_{0}$; or son terme général homogène d'ordre $\ell$ a une norme égale à $o\left(2^{\ell}\right) \cdot\left\|Y_{0, \ell}\right\|$ où $\sum_{\ell} Y_{0, \ell}$ est le terme général d'une série convergente sur $\mathbb{B}$. Donc, $Y_{k}$ converge vers $Y_{0,0}$ uniformément sur tout compact d'une boule $\varepsilon_{0} \mathbb{B}$ suffisamment petite. Faisant décrire à $Y_{0,0}$ une base de $\mathfrak{g}_{0}$, et prenant pour $\varepsilon$ le plus petit des $\varepsilon_{0}$ correspondants, on a que $\mathfrak{g}_{0}(\varepsilon \mathbb{B}) \subset \mathfrak{h}(\varepsilon \mathbb{B}, 0)$. Ensuite, on prouve de même que $\mathfrak{g}_{1}\left(\varepsilon^{\prime} \mathbb{B}\right) \subset \mathfrak{h}\left(\frac{1}{2} \mathbb{B}, 0\right)$ pour $\varepsilon^{\prime}$ assez petit : partant cette fois-ci de $Y_{1,1} \in \mathfrak{g}_{1}$ et choisissant $Y_{1}=\sum_{i>0} Y_{1, i}$ dans $\mathfrak{h}(\mathbb{B})$ on pose cette fois-ci

$$
Y_{k}=\frac{k+1}{k} Y_{k-1}-\left[X, Y_{k-1}\right]=Y_{0,1}+\sum_{\ell>k}(-1)^{k} C_{\ell-2}^{k} Y_{1, \ell} .
$$

La conclusion est ensuite la même. Appliquant maintenant la proposition 3.1.1 à $\mathfrak{h}\left(\varepsilon^{\prime \prime} \mathbb{B}, 0\right)$ avec $\varepsilon^{\prime \prime}=\inf \left(\varepsilon, \varepsilon^{\prime}\right)$ on obtient que $\mathfrak{h}\left(\varepsilon^{\prime \prime} \mathbb{B}, 0\right)$ contient tous les champs de vecteurs polynomiaux, et commes les polynômes sont denses dans les fonctions holomorphes sur la boule, la proposition est démontrée.

Donnons une conséquence utile du résultat précédent.

Proposition 3.1.3. - Si $z_{0} \in \mathbb{C}^{n}-\{0\}$ et si $U$ est un voisinage convenable de $z_{0}$, alors pour $p \geq 1$ la seule sous-algèbre de Lie fermée de $\mathfrak{g}(U)$ qui contient $\mathfrak{g}_{p}(U)$ est $\mathfrak{g}(U)$ elle même.

Preuve. - Prenons une carte affine $\left(x_{1}, \ldots, x_{n}\right)$ où $z_{0}$ a les coordonnées $(1, \ldots, 1)$. Ensuite, posons $y_{i}=1 / p-1 /\left(p x^{p}\right)$ pour définir une nouvelle carte $\left(y_{1}, \ldots, y_{n}\right)$ centrée en $z_{0}$. Dans les coordonnées $y_{i}$, le champ $x_{i}^{p+1} \partial / \partial x_{i}$ s'écrit $\partial / \partial y_{i}$. De plus, la sous-algèbre $\mathfrak{h}(U)$ de $\mathfrak{g}(U)$ engendrée par $\mathfrak{g}_{p}(U)$ contient $\mathfrak{g}_{k p}(U)$ pour tout $k \geq 1$ selon la proposition 3.1.1, d'où l'on tire qu'elle est stable pour la multiplication par $x_{i}^{p}$ pour tout $p$. Or, la fonction $x_{i}^{p}$ est équivalente à $1+p y$ à l'ordre 2 , d'où l'on tire facilement que $\mathfrak{h}(U)$ se surjecte sur

TOME $129-2001-\mathrm{N}^{\mathrm{O}} 2$ 
$\mathfrak{g}^{1}(U)$. Il ne reste plus qu'à appliquer la proposition 3.1.2 en notant au passage que $\mathfrak{g}(U)$ est engendrée par $\mathfrak{g}(U, 0)$ et les champs $\partial / \partial y_{i}$.

3.2. Quelques propriétés de $\mathfrak{g}_{\Gamma}$. - Pour $U$ ouvert dans $\mathbb{C}^{n}$, notons $G_{\Gamma}(U)$ l'ensemble des injections holomorphes $\phi$ de $U$ dans $\mathbb{C}^{n}$ telles qu'il existe une suite $\gamma_{n} \in \Gamma$ convergeant uniformément vers $\phi$ sur tout compact de $U$. Par convention, on suppose que l'identité en tant qu'élément de $\Gamma$ est définie partout; ainsi $G_{\Gamma}(U)$ n'est jamais vide. Les $G_{\Gamma}$ forment un préfaisceau, que nous ne chercherons pas à compléter en faisceau car ce faisant l'objet obtenu cesserait d'être un pseudogroupe ${ }^{(3)}$. Le germe en $z_{0}$ de $G_{\Gamma}$ est noté $G_{\Gamma}\left(z_{0}\right)$. De même, le germe en $z_{0}$ de $\mathfrak{g}_{\Gamma}$ est noté $\mathfrak{g}_{\Gamma}\left(z_{0}\right)$. Tout d'abord, le fait suivant découle immédiatement des définitions :

Lemme 3.2.1. - Pour tout $g \in G_{\Gamma}(U)$, on a $g^{*}\left(\mathfrak{g}_{\Gamma}(U)\right)=\mathfrak{g}_{\Gamma}(g(U))$.

Une autre conséquence facile des définitions est la suivante :

Proposition 3.2.2. - Soient $U$ un ouvert de $\mathbb{C}^{n}, X \in \mathfrak{g}_{\Gamma}(U), x_{0} \in U$ et $t_{0}$ un réel pour lequel le flot $\phi_{X}^{t_{0}}$ de $X$ au temps $t_{0}$ est défini au voisinage de $x_{0}$. Alors, il existe un voisinage $V$ de $x_{0}$ dans $U$ pour lequel $\phi_{X}^{t_{0}} \in G_{\Gamma}(V)$.

Preuve. - Considérons la portion $\mathcal{C}$ de la $X$-orbite de $x_{0}$ qui est paramétrée par les temps $t \in\left[0, t_{0}\right]$. C'est une courbe paramétrée compacte tracée dans $U$. Pour tout $x \in \mathcal{C}$, il existe un voisinage $U_{x}$ de $x$ dans $U$ sur lequel $X \in \mathfrak{g}_{\Gamma}^{\prime}\left(U_{x}\right)$. Alors par définition du préfaisceau $\mathfrak{g}^{\prime}$, il existe un voisinage ouvert $V_{x}$ de $x$ dans $U_{x}$ et un temps $\varepsilon_{x}>0$ pour lesquels chaque $\phi_{X}^{t}$ avec $t \in\left[0, \varepsilon_{x}\right]$ est limite uniforme sur $V_{x}$ d'une suite $\gamma_{n} \in \Gamma$. Par compacité de $\mathcal{C}$, on peut extraire de la famille $V_{x}$ un recouvrement fini $V_{1}, \ldots, V_{k}$. À chaque $V_{i}$ correspond un terme $\varepsilon_{i}$ de la famille $\varepsilon_{x}$. Soit $\varepsilon$ le minimum des $\varepsilon_{i}$; soit $I$ le plus grand sous-intervalle de $\left[0, t_{0}\right]$ sur lequel chaque $\phi_{X}^{t}$ est la limite au voisinage de $x_{0}$ d'une suite $\gamma_{n} \in \Gamma$ : on a d'abord $[0, \varepsilon] \subset I$; ensuite, si $t \in I \cap\left[0, t_{0}\left[\right.\right.$ alors $\phi_{X}^{t}\left(x_{0}\right)$ appartient à l'un $\operatorname{des} V_{i}$, et en le composant avec $\phi^{\varepsilon}$ on voit que $\left[0, \inf \left(t_{0}, t+\varepsilon\right)\right] \subset I$. De ceci découle facilement que $I=\left[0, t_{0}\right]$, ce qu'affirmait en somme la proposition.

Cette proposition éclaire le lien entre les théorèmes $\mathrm{A}$ et $\mathrm{C}$ :

Proposition 3.2.3. - Le point 2) du théorème $A$ est conséquence de son point 1) et du théorème $C$.

Preuve. - Soient $z_{0}, z_{1}$ et $\phi$ comme dans l'énoncé du théorème A. Selon le point 1) de ce théorème, il existe une petite boule pointée $\mathbb{B}^{*}$ incluse dans $\mathcal{B}$ car $\mathcal{B}$ est un voisinage épointé de l'origine. Par définition de $\mathcal{B}$ il existe aussi $\gamma_{0}$ et $\gamma_{1}$ dans $\Gamma$ tels que $\gamma_{0}\left(z_{0}\right)$ et $\gamma_{1}\left(z_{1}\right)$ appartiennent à $\mathbb{B}^{*}$. Soit $\mathcal{C}$ un arc de cercle

(3) Ceci parce que les fonctions du faisceau engendré par $G_{\Gamma}$ ne sont a priori que localement inversibles, et pas globalement inversibles. 
reliant $\gamma_{0}\left(z_{0}\right)$ à $\gamma_{1}\left(z_{1}\right)$ dans $\mathbb{B}^{*}$ : alors il existe un champ de vecteurs holomorphe dont $\mathcal{C}$ est un arc d'orbite, et le flot $\psi$ de ce champ pour un temps convenable vérifie $\psi\left(\gamma_{0}\left(z_{0}\right)\right)=\gamma_{1}\left(z_{1}\right)$. Alors par la proposition 3.2.2 et le théorème $\mathrm{C}$ on a $\phi \in G_{\Gamma}\left(\gamma_{0}\left(z_{0}\right)\right)$. Posons maintenant $\vartheta(z)=\psi^{-1} \gamma_{1} \phi \gamma_{0}^{-1}(z)$, en sorte que $\vartheta$ est un germe de fonction holomorphe défini en $\gamma_{0}\left(z_{0}\right)$ et fixant ce point. Considérons le flot radial $\zeta^{t}(z)=\mathrm{e}^{t}\left(z-\gamma_{0}\left(z_{0}\right)\right)+\gamma_{0}\left(z_{0}\right)$ : selon le théorème $\mathrm{C}$, $\zeta^{t} \in G_{\Gamma}\left(\gamma_{0}\left(z_{0}\right)\right)$ pour tout réel $t$; mais pour $t$ assez grand la partie linéaire de $\zeta^{t} \vartheta$ en $\gamma\left(z_{0}\right)$ est à la fois non-résonnante et dans le domaine de Poincaré, donc linéarisable et plongeable dans un flot, ce qui prouve que $\zeta^{t} \vartheta \in G_{\Gamma}\left(z_{0}\right)$ toujours par application du théorème C. En conclusion, $\phi=\gamma_{1}^{-1} \psi \zeta^{-t}\left(\zeta^{t} \vartheta\right) \gamma_{0}$ est composé d'éléments qui appartiennent tous au pseudogroupe $G_{\Gamma}$.

Par ailleurs, les résultats de la section 3.1 facilitent la preuve du théorème $\mathrm{C}$.

Proposition 3.2.4. - Pour que $\Gamma$ vérifie la conclusion du théorème $C$, il suffit que $\mathfrak{g}_{\Gamma}(0)$ contienne $\mathfrak{g}_{1}(0)$.

Preuve. - Soit $\left(X_{i}\right)$ une base de $\mathfrak{g}_{1}(0)$. Si $\mathfrak{g}_{\Gamma}(0)$ contient $\mathfrak{g}_{1}(0)$ alors pour chaque indice $i$ il existe un réel $\varepsilon_{i}>0$ pour lequel $X_{i} \in \mathfrak{g}_{\Gamma}\left(\varepsilon_{i} \mathbb{B}\right.$ ) (où $\mathbb{B}$ est la boule unité). Alors, pour $\varepsilon=\inf \left(\varepsilon_{i}\right)$ on a $\mathfrak{g}_{1}(\varepsilon \mathbb{B}) \subset \mathfrak{g}_{\Gamma}(\varepsilon \mathbb{B})$. Soit maintenant $z_{0} \in \mathcal{B}:$ par définition de $\mathcal{B}$ il existe $\gamma \in \Gamma$ tel que $\gamma\left(z_{0}\right) \in \varepsilon \mathbb{B}$, et selon la proposition 3.1.3 il existe une boule $\mathbb{B}_{0}$ de centre $\gamma\left(z_{0}\right)$ telle que $\mathfrak{g}_{\Gamma}\left(\mathbb{B}_{0}\right)=\mathfrak{g}\left(\mathbb{B}_{0}\right)$. Si cette boule est de surcroît assez petite, $\gamma^{-1}$ y est défini et on a alors $\mathfrak{g}_{\Gamma}\left(\gamma^{-1}\left(\mathbb{B}_{0}\right)\right)=\mathfrak{g}\left(\gamma^{-1}\left(\mathbb{B}_{0}\right)\right)$ en vertu du lemme 3.2.1. Pour résumer : quel que soit $z_{0}$ dans $\mathcal{B}$, les germes en $z_{0}$ de $\mathfrak{g}$ et de $\mathfrak{g}_{\Gamma}$ sont identiques, ce qui est bien la conclusion du théorème $\mathrm{C}$.

3.3. Construction d'éléments de $\mathfrak{g}_{\Gamma}$. - Maintenant, nous montrons comment construire des éléments de $\mathfrak{g}_{\Gamma}(U)$ pour tout ouvert $U$ de $\mathbb{C}^{n}$. Soit $\gamma_{n}$ une suite d'éléments de $\Gamma$ qui sont tous définis sur $U$ et tendent uniformément vers l'identité sur ce domaine. On peut alors trouver une suite d'entiers $p_{n}$ tendant vers $+\infty$ et telle que

$$
\sup _{n \in \mathbb{N}}\left(p_{n} \cdot \sup _{z \in U}\left|\gamma_{n}(z)-z\right|\right)<+\infty
$$

La fonction $Z_{n}(z)=p_{n}\left(\gamma_{n}(z)-z\right)$ peut être vue formellement comme un élément de $\mathfrak{g}(U)$, car elle associe au point $z$ le "vecteur" $p_{n}\left(\gamma_{n}(z)-z\right)$. De ce point de vue, $Z_{n}$ est une famille normale donc, quitte à passer à une soussuite convenable, converge sur $U$ vers un champ de vecteurs $Z$ nul en zéro et uniformément borné sur $U$.

Proposition 3.3.1. — Dans le contexte précédent, on a $Z \in \mathfrak{g}_{\Gamma}^{\prime}(U)$.

TOME $129-2001-\mathrm{N}^{\mathrm{O}} 2$ 
Preuve. - Donnons-nous $\varepsilon>0, K$ compact dans $U$ et $t_{0} \in I_{K}$. Soit $V$ un voisinage compact dans $U$ de l'image de $K \times\left[0, t_{0}\right]$ par le flot de $Z$. Soit

$$
\kappa=\sup _{V \times V} \frac{|Z(x)-Z(y)|}{|x-y|} .
$$

Lorsque $n$ est assez grand, on a la majoration

$$
\sup _{z \in V}\left|\gamma_{n}(z)-z-p_{n}^{-1} Z(z)\right| \leq p_{n}^{-1} \varepsilon
$$

car $p_{n}\left(\gamma_{n}(z)-z\right)$ tend vers $Z$ uniformément sur $V$. Approchons $t_{0}$ par une suite de rationnels de la forme $r_{n} / p_{n}$ avec $r_{n}$ entier. La méthode des lignes polygonales d'Euler pour la construction des flots affirme que :

- pour $n$ assez grand et $z_{0} \in K$, la suite définie par induction en posant $z_{i+1}=z_{i}+p_{n}^{-1} Z\left(z_{i}\right)$ est bien définie pour $i \leq r_{n}$ et entièrement contenue dans $V$;

- lorsque $n$ tend vers $+\infty$, la fonction qui à $z_{0}$ associe $z_{r_{n}}$ tend uniformément sur $K$ vers $\phi_{Z}^{t_{0}}$.

Posons maintenant par induction, tant que la formule garde un sens

$$
z_{0}^{\prime}=z_{0}, \quad z_{i+1}^{\prime}=\gamma_{n}\left(z_{i}^{\prime}\right) .
$$

On tire de (4), sous réserve de l'existence des termes en " $z^{\prime \prime}$ "

$$
\begin{aligned}
\left|z_{i+1}^{\prime}-z_{i+1}\right| & =\left|\gamma_{n}\left(z_{i}^{\prime}\right)-z_{i}-p_{n}^{-1} Z\left(z_{i}\right)\right| \\
& =\left|\gamma_{n}\left(z_{i}^{\prime}\right)-z_{i}^{\prime}-p_{n}^{-1} Z\left(z_{i}^{\prime}\right)+\left(z_{i}^{\prime}-z_{i}\right)+p_{n}^{-1}\left(Z\left(z_{i}^{\prime}\right)-Z\left(z_{i}\right)\right)\right| \\
& \leq\left|\gamma_{n}\left(z_{i}^{\prime}\right)-z_{i}^{\prime}-p_{n}^{-1} Z\left(z_{i}^{\prime}\right)\right|+\left|z_{i}^{\prime}-z_{i}\right|+p_{n}^{-1}\left|Z\left(z_{i}^{\prime}\right)-Z\left(z_{i}\right)\right| \\
& \leq p_{n}^{-1} \varepsilon+\left|z_{i}^{\prime}-z_{i}\right|\left(1+p_{n}^{-1} \kappa\right)
\end{aligned}
$$

d'où par récurrence (toujours sous réserve d'existence de $z_{i}^{\prime}$ ) :

$$
\left|z_{i}^{\prime}-z_{i}\right| \leq p_{n}^{-1} \varepsilon \sum_{k=0}^{i}\left(1+p_{n}^{-1} \kappa\right)^{k} .
$$

La suite $r_{n} / p_{n}$ tendant vers $t_{0}$, il existe une constante entière $c$ telle que $r_{n}<c p_{n}$, et pour $i \leq c p_{n}$, la majoration (5) devient

$$
\left|z_{i}^{\prime}-z_{i}\right| \leq p_{n}^{-1} \varepsilon\left[i\left(1+p_{n}^{-1} \kappa\right)^{i}\right] \leq p_{n}^{-1} \varepsilon\left(c p_{n} \mathrm{e}^{\kappa c}\right)
$$

puisque pour $\alpha \beta \geq 0$, on a $(1+\alpha)^{\beta}=\mathrm{e}^{\beta \operatorname{Ln}(1+\alpha)} \leq \mathrm{e}^{\alpha \beta}$. On peut donc écrire

$$
\left|z_{i}^{\prime}-z_{i}\right| \leq c \mathrm{e}^{c \kappa} \varepsilon
$$

ceci uniformément sur $K$. On en déduit par induction que pour $\varepsilon$ assez petit et $n$ assez grand, la suite $z_{i}^{\prime}$ est définie pour $i \leq r_{n}$, car en vertu de (6) ceux de ses termes d'indice $i \leq r_{n}$ qui sont définis sont dans $V$, ce qui permet pour chacun d'entre eux de définir le suivant. Ensuite, cette majoration montre que la fonction qui à $z_{0}$ associe $z_{r_{n}}^{\prime}$ tend uniformément sur $K$ vers le flot de $Z$ au 
temps $t_{0}$ (elle est uniformément de plus en plus proche d'une fonction qui tend elle-même uniformément vers ce flot).

L'exemple (en une variable) de $\gamma_{k}(z)=z+z^{k}$ montre que l'on peut avoir $Z=0$ même si aucun des termes de la suite $\gamma_{k}$ n'est l'identité. Il faut donc choisir soigneusement la suite $\gamma_{k}$ pour utiliser la proposition 3.3.1. Nous nous baserons sur l'existence d'une homothétie dans l'adhérence du groupe des parties linéaires d'éléments de $\Gamma$. Nous disposons maintenant d'assez de matériel pour passer à la preuve des résultats annoncés.

\section{Preuve des théorèmes $\mathrm{A}$ et $\mathrm{C}$}

On suppose désormais que $\Gamma$ vérifie les hypothèses du théorème $A$ pour les ouverts $Z$ et $V$ définis en 2.4.1.

\section{1. $\mathcal{B}$ est un voisinage ouvert épointé de 0 (point $1 \mathrm{du}$ théorème $\mathrm{A}$ )}

Preuve. - En vertu de la proposition 2.4.2, il existe des éléments de $\Gamma$ dont la partie linéaire est arbitrairement proche d'une homothétie de rapport $\lambda$ avec $|\lambda| \neq 1$. La dynamique d'un tel élément $\gamma$ fait que $\mathcal{B}$ est un voisinage épointé de l'origine : pour $\varepsilon$ assez petit, pour tout $x$ tel que $0<|x|<\varepsilon$, la suite $\gamma^{n}(x)$ tend vers l'origine. Ceci étant, soit $y \in \mathcal{B}:$ par définition de $\mathcal{B}$ il existe $\gamma_{0} \in \Gamma$ tel que $\left|\gamma_{0}(y)\right|<\varepsilon$. Alors, on a encore $\left|\gamma_{0}(z)\right|<\varepsilon$ pour tout $z$ assez proche de $y$, donc $\gamma^{n} \gamma_{0}(z)$ tend vers 0 , ce qui prouve que $z \in \mathcal{B}$. Puisque $\mathcal{B}$ contient tous les points $z$ voisins de chacun de ses points $y$, c'est un ouvert.

4.2. Réduction de la preuve. - Nous venons de montrer le point $1 \mathrm{du}$ théorème $\mathrm{A}$, donc le second point de ce théorème est conséquence du théorème $\mathrm{C}$ en vertu de la proposition 3.2.3. D'autre part, la proposition 3.2.4 permet de se ramener à construire dans $\mathfrak{g}_{\Gamma}(0)$ une base de $\mathfrak{g}_{1}(0)$. Ceci étant, supposons construit un champ linéaire $\ell$ non trivial dans $\mathfrak{g}_{0}(0) \cap \mathfrak{g}_{\Gamma}(0)$ : alors, la proposition 2.3.2 implique que $\mathfrak{g}_{\Gamma}(0)$ se surjecte sur $\mathfrak{s g}^{1}(0)$. Soit alors $\gamma \in \Gamma$, un élément dont le déterminant n'est pas de module 1 : on peut donc écrire $\gamma(z)=\lambda \gamma_{0}(z)$ où $|\lambda| \neq 1$ et $\operatorname{det}\left(\gamma_{0}\right)=1$; quitte à remplacer $\gamma$ par son inverse on peut supposer $|\lambda|<1$. Mais puisque $\mathfrak{g}_{\Gamma}(0)$ se surjecte sur $\mathfrak{s g}^{1}(0)$, il existe un élément $\gamma_{1}$ de $G_{\Gamma}(0)$ dont la partie linéaire est $\gamma_{0}^{-1}$, aussi $\gamma_{0}$ contient $\gamma_{2}=\gamma \gamma_{1}$ dont la partie linéaire est l'homothétie de rapport $\lambda$. Cet élément est linéarisable au voisinage de 0 . Effectuons cette linéarisation, soit $X_{1} \in \mathfrak{g}_{1}-\{0\}$, $X=X_{1}+X_{2}+\cdots \in \mathfrak{g}_{\Gamma}(0)$ (qui existe parce que $\mathfrak{g}_{\Gamma}(0)$ se surjecte sur $\mathfrak{s g}^{1}(0)$ ) et soit $\mathbb{B}$ une petite boule centrée en 0 qui est dans le domaine de définition commun à $\gamma_{2}$ et $X$. Un calcul évident donne $\lim _{k \rightarrow+\infty} \lambda^{-k}\left(\gamma_{2}^{k}\right)^{*}(X)=X_{1}$. En appliquant ce procédé à une base de $\mathfrak{g}_{1}(0)$, on voit donc que si $\mathfrak{g}_{0}(0) \cap \mathfrak{g}_{\Gamma}(0)$

TOME $129-2001-\mathrm{N}^{\mathrm{O}} 2$ 
contient un champ linéaire, ce germe contient aussi $\mathfrak{g}_{1}(0)$, ce qui ramène en fin de compte la preuve des théorèmes $\mathrm{A}$ et $\mathrm{C}$ à celle de la proposition suivante,

Proposition 4.2.1. - $\mathfrak{g}_{\Gamma}(0)$ contient un champ linéaire non-nul.

Comme la preuve de cette proposition est un peu technique, nous en donnons d'abord l'idée.

Si nous savions déjà que $G_{\Gamma}(0)$ contient un germe d'homothétie dilatante $h$, nous pourrions choisir n'importe quel $\gamma_{0}$ dans $\Gamma$ et poser par récurrence $\gamma_{n+1}=$ $h \gamma_{n} h^{-1}$ : cette suite convergeant vers la partie linéaire de $\gamma_{0}$ sur tout le domaine $\mathbb{D}$ de $h$, on en déduirait que $G_{\Gamma}(\mathbb{D})$ contient les parties linéaires de tous les éléments de $\Gamma$ et on concluerait par densité de $\Gamma$ dans $\operatorname{SL}(n, \mathbb{C})$ sans même utiliser la proposition 3.3.1.

Malheureusement, tout ce que nous savons, c'est que $\Gamma$ contient un $\gamma$ proche d'une homothétie $h$. L'algorithme précédent ne va plus pour ce $\gamma$, car si l'on pose $\gamma_{n+1}=\gamma \gamma_{n} \gamma^{-1}$, alors le terme linéaire de $\gamma_{n}$ diverge si celui de $\gamma_{0}$ est générique dans $\operatorname{GL}(n, \mathbb{C})$ et si celui de $\gamma$ n'est pas une homothétie.

Il faut donc combiner la conjugaison par $\gamma$ avec une deuxième opération qui ramène la partie linéaire de $\gamma_{n}$ au voisinage de 1 sans pour autant en réeloigner les termes d'ordre supérieur. Mais puisque la partie linéaire de $h \gamma_{0} h^{-1} \gamma_{0}^{-1}$ est triviale pour tout $\gamma_{0}$, on peut imaginer que l'opération de crochet avec $\gamma$ remplit le but fixé. C'est effectivement le cas, et la suite $\gamma_{n+1}=\gamma\left[\gamma, \gamma_{n}\right] \gamma^{-1}$ tend vers l'identité, au moins au sens des séries formelles en 0.

Maintenant, on veut bien sûr conclure en utilisant la proposition 3.3.1, et le problème est donc de vérifier : a) qu'il existe un domaine $\mathbb{B}$ sur lequel $\gamma_{n}$ est toujours définie et converge uniformément vers l'identité; et b) que la suite des parties linéaires de $\gamma_{n}$ tend vers l'identité strictement moins vite que la suite des termes résiduels (c'est à ce prix que le champ $Z$ de 3.3.1 sera linéaire non-nul). Mais ceci ne peut pas toujours être vrai (c'est faux si $\gamma$ est une homothétie, ou si les parties linéaires de $\gamma$ et $\gamma_{0}$ commutent). On est donc conduit à supposer que $\gamma$, bien que proche d'une homothétie, n'en est pas une, et que $\gamma_{0}$ n'est pas quelconque ${ }^{(4)}$.

Passons maintenant à la preuve proprement dite. Selon la proposition 2.4.1, il existe un $\gamma \in \Gamma$ dont la partie linéaire a des valeurs propres $\lambda_{1}, \ldots, \lambda_{n}$ qui vérifient

$$
1<\left|\lambda_{1}\right|<\cdots<\left|\lambda_{n}\right|<\left|\lambda_{1}\right|^{2}
$$

ce qui implique que $\gamma$ est linéarisable au voisinage de l'origine puisque sa partie linéaire est non-résonante et dans le domaine de Poincaré ( $c f$. [1], p. 72). On se place pour la suite dans une carte locale où $\gamma$ est linéaire et défini sur $\mathbb{B}$.

(4) Il faut signaler que cette méthode est une version simplifiée d'un algorithme mis en œuvre par S. Lamy dans sa thèse (voir [9], preuve du théorème 3.1). 
Soit maintenant $\gamma_{0} \in \Gamma$ et posons par récurrence $\gamma_{k+1}=\gamma\left[\gamma, \gamma_{k}\right] \gamma^{-1}$. Posons $\gamma_{k}=L_{k} \phi_{k}$ où $L_{k}$ est la partie linéaire de $\gamma_{k}$ et $\phi_{k}=L_{k}^{-1} \gamma_{k}$ est supposé défini sur le même domaine que $\gamma_{k}$ (formellement, on considère que $L_{k}$, qui est linéaire, est défini partout). Un calcul direct donne

$$
L_{k+1}=\gamma\left[\gamma, L_{k}\right] \gamma^{-1}, \quad \phi_{k+1}=\gamma L_{k}\left[\gamma, \phi_{k}\right] L_{k}^{-1} \gamma^{-1} \text {. }
$$

Nous étudions les suites $L_{k}$ et $\phi_{k}$; nous montrons que si $\gamma_{0}$ est bien choisi, la suite $\gamma_{k}$ tend vers l'identité uniformément sur $\mathbb{B}$ et montrons que le champ $Z$ qui lui est alors associé par la proposition 3.3.1 est linéaire.

Remarque 4.2.2. - Par hypothèse on peut choisir $L_{0}$ aussi proche que l'on veut de toute matrice de $\operatorname{SL}(n, \mathbb{C})$ donnée d'avance. Ensuite, quitte à renormaliser notre carte locale (ce qui influe sur $\phi_{0}$ sans changer ni $\gamma$ ni $L_{0}$ ), on peut supposer :

- que $\phi_{0}$ est défini sur $\mathbb{B}$;

- que sur $\mathbb{B}$ on a $\left|\phi_{0}(z)-z\right|<\varepsilon|z|^{2}$ où $\varepsilon>0$ est une constante que l'on s'est donnée d'avance.

À partir de là, nous procédons en quatre étapes : nous étudions d'abord le comportement de $L_{k}$, puis celui (qui en dépend) de $\phi_{k}$, puis nous comparons les vitesses de convergence de ces deux suites avant de conclure.

4.3. Étude de la suite $\boldsymbol{L}_{\boldsymbol{k}}$. - Rappelons un fait classique. Si $x \mapsto f(x)$ est une fonction de classe $C^{1}$ définie au voisinage de 0 , de $\mathbb{R}^{n}$ dans $\mathbb{R}^{n}$, telle que $f(0)=0$ et telle que $\|d f(0)\|<1$ (où $\|\cdot\|$ est la norme Sup sur les matrices) alors, pour $x_{0}$ appartenant à un ouvert dense d'un voisinage de 0 , la suite récurrente $x_{k+1}=f\left(x_{k}\right)$ tend vers 0 avec l'estimation asymptotique $(\rho-\varepsilon)^{k} \leq$ $\left\|x_{k}\right\| \leq(\rho+\varepsilon)^{k}$ où $\rho$ est le rayon spectral de $d f(0)$.

Posons maintenant $L_{k}=\mathrm{Id}+l_{k}$. Si $\left|\ell_{k}\right|<1$ alors on peut écrire $L_{k}^{-1}$ comme une somme infinie $L_{k}^{-1}=\mathrm{Id}-\ell_{k}+\ell_{k}^{2}-\ell_{k}^{3}+\cdots$, de sorte que

$$
\ell_{k+1}=\gamma^{2} \ell_{k} \gamma^{-2}-\gamma \ell_{k} \gamma^{-1}+o\left(\ell_{k}\right)
$$

comme il résulte d'un calcul direct à partir de la formule (7). La transformation linéaire $\gamma^{2} \ell_{k} \gamma^{-2}-\gamma \ell_{k} \gamma^{-1}$ est donc la différentielle en l'identité de l'application $L_{k} \rightarrow L_{k+1}$, de $\operatorname{GL}(n, \mathbb{C})$ dans lui-même, et on a tôt fait de calculer ses valeurs propres. On voit que c'est une contraction diagonalisable de plus grande valeur propre le nombre $\rho$ défini ci-dessous, formule (8). On en déduit sans peine le comportement asymptotique de la suite $L_{k}$ en fonction de son premier terme :

FAIT 4.3.1. - Si $L_{0}$ est générique dans un voisinage assez petit de l'identité, alors la suite $L_{k}$ converge vers l'identité et on a l'estimation suivante sur sa norme,

$$
(\rho-\varepsilon)^{k} \leq \sup _{z \in \mathbb{C}^{n}} \frac{\left|L_{k}(z)-z\right|}{|z|} \leq(\rho+\varepsilon)^{k}
$$

TOME $129-2001-\mathrm{N}^{\mathrm{O}} 2$ 
où $\varepsilon>0$ est aussi petit que l'on veut et où $\rho$ est le nombre

$$
\rho=\max \left\{\left|\left(\frac{\lambda_{i}}{\lambda_{j}}\right)^{2}-\frac{\lambda_{i}}{\lambda_{j}}\right|\right\}
$$

4.4. Étude de la suite $\phi_{\boldsymbol{k}}$. — À l'aide de l'hypothèse (a) de 4.2.2, nous pouvons supposer $\phi_{0}$ définie sur $\mathbb{B}$. Nous définissons les suites

$$
\varepsilon_{k}=\sup _{z \in \mathbb{C}^{n}} \frac{\left|L_{k}(z)-z\right|}{|z|}, \quad \varepsilon_{k}^{\prime}=\sup _{z \in \mathbb{B}} \frac{\left|\phi_{k}(z)-z\right|}{\left|z^{2}\right|} .
$$

A priori $\varepsilon_{k}^{\prime}$ n'est pas toujours bien défini. Toutefois, l'hypothèse (b) nous permet de supposer $\varepsilon_{0}$ défini et aussi petit que l'on veut.

Ceci étant, en notant $w=\phi_{k}(z)$, on tire de $(9):|w| \geq|z|-\varepsilon_{k}^{\prime}|z|^{2} \geq\left(1-\varepsilon_{k}^{\prime}\right)|z|$ pour $z \in \mathbb{B}$; si $\varepsilon_{k}^{\prime}<1$, ceci implique que $\phi_{k}$ est inversible sur $\mathbb{B}, \phi_{k}^{-1}$ définie sur $\left(1-\varepsilon_{k}^{\prime}\right) \mathbb{B}$ et que

$$
\sup _{w \in\left(1-\varepsilon_{k}^{\prime}\right) \mathbb{B}} \frac{\left|\phi_{k}^{-1}(w)-w\right|}{|w|^{2}} \leq \frac{\varepsilon_{k}^{\prime}}{\left(1-\varepsilon_{k}^{\prime}\right)^{2}} .
$$

Alors, pour $z \in \mathbb{B}: L_{k}^{-1} \gamma^{-1}(z) \in\left(1-\varepsilon_{k}\right)^{-1}\left|\lambda_{1}^{-1}\right| \mathbb{B}\left(\right.$ le " $\left(1-\varepsilon_{k}\right)^{-1}$ " est un majorant de la norme de $L_{k}^{-1}$ découlant directement de (9)). De ce fait, $\phi_{k}^{-1} L_{k}^{-1} \gamma^{-1}(z)$ est défini sur tout $\mathbb{B}$, et en appliquant (9) on obtient

$$
\sup _{z \in \mathbb{B}}\left|\phi_{k}^{-1} L_{k}^{-1} \gamma^{-1}(z)-L_{k}^{-1} \gamma^{-1}(z)\right| \leq \frac{\varepsilon_{k}^{\prime}}{\left(1-\varepsilon_{k}\right)^{2}\left(1-\varepsilon_{k}^{\prime}\right)^{2}}\left|\lambda_{1}\right|^{-2}
$$

Ensuite, comme $\gamma$ est linéaire, diagonale et de valeurs propres dont le module majore $\left|\lambda_{1}\right|$, on a

$$
\sup _{z \in \mathbb{B}}\left|\gamma^{-1} \phi_{k}^{-1} L_{k}^{-1} \gamma^{-1}(z)-\gamma^{-1} L_{k}^{-1} \gamma^{-1}(z)\right| \leq \frac{\varepsilon_{k}^{\prime}}{\left(1-\varepsilon_{k}^{\prime}\right)^{2}\left(1-\varepsilon_{k}\right)^{2}}\left|\lambda_{1}\right|^{-3}
$$

Appliquant $\phi_{k}$ au premier terme $w=\gamma^{-1} \phi_{k}^{-1} L_{k}^{-1} \gamma^{-1}(z)$ de (10), qui vérifie quant à lui ${ }^{(5)}$

$$
|w| \leq \frac{1}{\left(1-\varepsilon_{k}\right)\left(1-\varepsilon_{k}^{\prime}\right)}\left|\lambda_{1}\right|^{-2}|z|
$$

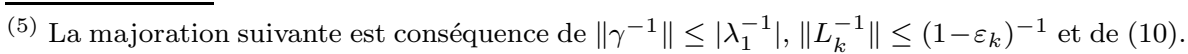
BULLETIN DE LA SOCIÉTÉ MATHÉMATIQUE DE FRANCE 
on tire

$$
\begin{aligned}
\mid \phi_{k} \gamma^{-1} & \phi_{k}^{-1} L_{k}^{-1} \gamma^{-1}(z)-\gamma^{-1} L_{k}^{-1} \gamma^{-1}(z) \mid \\
& =\left|\phi_{k}(w)-w+w-\gamma^{-1} L_{k}^{-1} \gamma^{-1}(z)\right| \\
& =\left|\phi_{k}(w)-w+\gamma^{-1} \phi_{k}^{-1} L_{k}^{-1} \gamma^{-1}(z)-\gamma^{-1} L^{-1} \gamma^{-1}(z)\right| \\
& \leq\left|\phi_{k}(w)-w\right|+\left|\gamma^{-1} \phi_{k}^{-1} L_{k}^{-1} \gamma^{-1}(z)-\gamma^{-1} L_{k}^{-1} \gamma^{-1}(z)\right| \\
& \leq \varepsilon_{k}^{\prime}\left(\frac{1}{\left(1-\varepsilon_{k}\right)\left(1-\varepsilon_{k}^{\prime}\right)}\left|\lambda_{1}\right|^{-2}\right)^{2}+\frac{\varepsilon_{k}^{\prime}}{\left(1-\varepsilon_{k}\right)^{2}\left(1-\varepsilon_{k}\right)^{2}}\left|\lambda_{1}\right|^{-3}
\end{aligned}
$$

puis en appliquant $\gamma L_{k} \gamma$ et en majorant la norme de cette transformation linéaire

$\sup _{z \in \mathbb{B}}\left|\gamma L_{k} \gamma \phi_{k} \gamma^{-1} \phi_{k}^{-1} L_{k}^{-1} \gamma^{-1}(z)-z\right| \leq \frac{\left(1+\varepsilon_{k}\right) \varepsilon_{k}^{\prime}}{\left(1-\varepsilon_{k}\right)^{2}\left(1-\varepsilon_{k}^{\prime}\right)^{2}}\left(\left|\lambda_{1}\right|^{-4}+\left|\lambda_{1}\right|^{-3}\right)\left|\lambda_{n}\right|^{2}$.

Le nombre $\rho^{\prime}=\left(\left(\left|\lambda_{1}\right|^{-4}+\left|\lambda_{1}\right|^{-3}\right)\left|\lambda_{n}\right|^{2}\right.$ est proche de 0 si $\left|\lambda_{1}\right|$ et $\left|\lambda_{n}\right|$ sont à la fois grands et voisins. Alors, si $\varepsilon_{0}$ et $\varepsilon_{0}^{\prime}$ sont assez petits, on a par induction

$$
\varepsilon_{k+1}^{\prime}=\sup _{z \in \mathbb{B}}\left|\gamma L_{k} \gamma \phi_{k} \gamma^{-1} L_{k}^{-1} \gamma^{-1}(z)-z\right| \leq\left(\rho^{\prime}+\varepsilon\right) \varepsilon_{k}
$$

où $\rho^{\prime}+\varepsilon<1$. Ceci montre que la suite $\phi_{k}$ reste définie sur $\mathbb{B}$ et y converge uniformément vers l'identité à vitesse exponentielle.

4.5. Comparaison de $\rho$ et $\rho^{\prime}$. - Dans l'adhérence du groupe des parties linéaires d'éléments de $\Gamma$, il y a $\operatorname{SL}(n, \mathbb{C})$ et la matrice d'une homothétie dont le rapport $\lambda$ a un module autre que 1 . Il y a donc aussi la famille $M(k, t)$ des matrices diagonales de valeurs propres

$$
\lambda_{1}=\cdots=\lambda_{n-1}=\lambda^{k} t^{-1}, \quad \lambda_{n}=\lambda^{k} t^{n-1} \quad(t>0) .
$$

Les nombres $\rho$ et $\rho^{\prime}$ correspondant à $M(k, t)$ sont, pour $\lambda^{k} \gg t \gg 1$ :

$$
\rho=t^{2 n}-t^{n}, \quad \rho^{\prime}=t^{2 n}\left(|\lambda|^{2 k}+|\lambda|^{k} t\right)^{-1} .
$$

On voit donc que pour $\left|\lambda^{k}\right| \gg t^{n} \gg 1$, on a $\rho>\rho^{\prime}$. En choisissant $\gamma$ proche d'une matrice de ce type, nous pouvons supposer dans l'étude précédente que $\phi_{k}$ converge vers l'identité plus vite que $L_{k}, \phi_{k}-\mathrm{Id}=o\left(L_{k}-\mathrm{Id}\right)$ sur $\mathbb{B}$.

4.6. Conclusion de la preuve. - Soit $p_{k}=\left\|L_{k}-\mathrm{Id}\right\|^{-1}$. On a

$$
\begin{aligned}
p_{k}\left(\gamma_{k}(z)-z\right) & =p_{k}\left(L_{k}(z)-z\right)+p_{k}\left(L_{k}(z) \circ\left(\phi_{k}(z)-\mathrm{Id}\right)\right) \\
& =p_{k}\left[\left(L_{k}(z)-z\right)+o\left(L_{k}(z)-z\right)\right]
\end{aligned}
$$

d'où l'on tire en appliquant la proposition 3.3.1 que $\mathfrak{g}_{\Gamma}(\mathbb{B})$ contient tous les champs linéaires qui sont valeurs d'adhérence de la famille $L_{k}(z)-z$. Ces champs doivent être dans $\mathfrak{s l}(n, \mathbb{C})$ puisque, pour $k>0$,

$$
\operatorname{det}\left(L_{k}\right)=\operatorname{det}\left(\gamma^{2} L_{k-1} \gamma^{-1} L_{k-1}^{-1} \gamma^{-1}\right)=1
$$

TOME $129-2001-\mathrm{N}^{\mathrm{O}} 2$ 


\section{Preuve du théorème $D$}

Dans ce paragraphe, on ne prétend pas à l'originalité; la méthode employée s'inspire de résultats anciens de Cartan et aliis sur les tissus, et le lien avec le théorème fondamental de la géométrie projective complexe deviendra vite flagrant ([4], $\left.\mathrm{n}^{0} 10\right)$.

Soient $\Gamma$ et $\Gamma^{\prime}$ deux pseudogroupes vérifiant les hypothèses du théorème $\mathrm{A}$. Soit $\phi$ un germe de bijection bicontinue, que nous pouvons supposer défini sur la boule unité $\mathbb{B}$, elle-même contenue dans $\mathcal{B}$ (quitte à renormaliser). Supposons enfin que $\phi$ conjugue $\Gamma$ à $\Gamma^{\prime}$ : pour tout $\gamma \in \Gamma$ et tout $\gamma^{\prime} \in \Gamma^{\prime}, \phi \circ \gamma \circ \phi^{-1}$ est dans $\Gamma^{\prime}$ et $\phi^{-1} \circ \gamma^{\prime} \circ \phi$ dans $\Gamma$. Nous commençons par nous rappeler que si une fonction continue $\psi$ est holomorphe sur $\mathbb{B}-\{0\}$, alors par le théorème de Hartogs $\psi$ possède un prolongement holomorphe à $\mathbb{B}$ et celui-ci ne peut être que $\psi$ : donc, pour prouver que $\phi$ est holomorphe sur $\mathbb{B}$, il suffit de montrer que $\phi$ est holomorphe en tout point $z_{0}$ de $\mathbb{B}-\{0\}$. L'argument fonctionne mutatis mutandis pour les fonctions antiholomorphes.

Fixons $z_{0} \in \mathbb{B}-\{0\}$ et un système de coordonnées affines $\left(x_{1}, \ldots, x_{n}\right)$ d'origine $z_{0}$; nous notons $\pi_{1}, \ldots, \pi_{n}$ les $n$ projecteurs canoniques de l'espace sur les axes de ce système (ainsi, par exemple, $\pi_{1}\left(x_{1}, x_{2}, \ldots, x_{n}\right)=\left(x_{1}, 0, \ldots, 0\right)$ ). Nous posons aussi $\pi_{0}\left(x_{1}, \ldots, x_{n}\right)=\left(x_{1}+\cdots+x_{n}, \ldots, x_{1}+\cdots+x_{n}\right)$. Ensuite, considérons la fonction linéaire $f\left(x_{1}, x_{2}, \ldots, x_{n}\right)=\left(x_{1}, \frac{1}{2} x_{2}, \ldots, \frac{1}{2} x_{n}\right):$ selon le théorème $\mathrm{A}$, il existe un voisinage $V_{1}$ de $z_{0}$ sur lequel la fonction $f$ est limite uniforme d'une suite $\gamma_{n}$ de $\Gamma$. Alors, comme $\phi$ conjugue $\Gamma$ à $\Gamma^{\prime}$ il existera un voisinage $V_{1}^{\prime}$ de $z_{0}^{\prime}=\phi\left(z_{0}\right)$ sur lequel la suite $\phi \circ \gamma_{n} \circ \phi^{-1}$ d'éléments de $\Gamma^{\prime}$ converge uniformément vers une fonction holomorphe $f^{\prime}$. De même, comme la suite $f^{n}$ des itérés positifs de $f$ converge uniformément sur $V_{1}$ vers $\pi_{1}$ lorsque $V_{1}$ est bien choisi (par exemple convexe), la suite $f^{\prime n}$ convergera sur $V_{1}^{\prime}$ vers une fonction holomorphe $\pi_{1}^{\prime}$ qui a en commun avec $\pi_{1}$ la propriété topologique de ne pas être constante, mais d'être cependant constante le long des feuilles d'un feuilletage topologique régulier $\mathcal{F}_{1}^{\prime}$ de codimension réelle 2 (c'est l'image par $\phi$ du feuilletage $\mathcal{F}_{1}$ par hyperplans d'équation $\left.d x_{1}=0\right)$. Par le même procédé on peut construire les autres projecteurs $\pi_{i}^{\prime}(0 \leq i \leq n)$ comme limite uniforme de suites $\gamma_{n} \in \Gamma$ sur des voisinages $V_{i}$ de $z_{0}$, et en déduire l'existence de domaines $V_{i}^{\prime}$, de feuilletages $\mathcal{F}_{i}^{\prime}$ et de fonctions $\pi_{i}^{\prime}$ leur correspondant. Nous allons maintenant montrer que $\phi$ est holomorphe ou anti-holomorphe sur l'intersection $V$ des $V_{i}(0 \leq i \leq n)$.

LEMME 5.1. - Les feuilletages $\mathcal{F}_{i}^{\prime}$ sont des feuilletages holomorphes; de plus ils sont en position générale (au sens où en tout point, leurs espaces tangents sont en position générale en tant qu'hyperplans de $\left.\mathbb{C}^{n}\right)$.

Preuve. - $\mathcal{F}_{i}^{\prime}$ est défini par l'équation $d \pi_{i}^{\prime}=0$ et $\pi_{i}^{\prime}$ est une fonction holomorphe, donc $\mathcal{F}_{i}^{\prime}$ est un feuilletage holomorphe. Ce feuilletage est a priori singulier; toutefois, en vertu du théorème $\mathrm{A}$, il existe pour tout couple $z_{1}, z_{2}$ 
de points de $V$ un voisinage $W$ de $z_{1}$ et une suite $\gamma_{n} \in \Gamma$ tendant vers la translation $\tau(z)=z+z_{2}-z_{1}$ uniformément sur $W$. Il existe une autre translation $\tau_{i}$ telle que $\pi_{i} \circ \tau=\tau_{i} \circ \pi_{i}$, et elle est aussi limite d'une suite $\gamma_{i, n}$ uniformément au voisinage de $\pi_{i}\left(z_{1}\right)$. Alors, $\phi \circ \gamma_{n} \circ \phi^{-1}$ converge vers une fonction holomorphe $\tau^{\prime}$ définie au voisinage de $\phi\left(z_{1}\right)$ et telle que $\tau^{\prime}\left(\phi\left(z_{1}\right)\right)=\phi\left(z_{2}\right)$; de même $\phi \circ \gamma_{i, n} \circ \phi^{-1}$ converge vers une fonction holomorphe $\tau_{i}^{\prime}$ définie au voisinage de $\pi_{i}^{\prime}\left(\phi\left(z_{1}\right)\right)$; on a $\pi_{i}^{\prime} \circ \tau^{\prime}=\tau_{i}^{\prime} \circ \pi_{i}^{\prime}$ et comme la composition avec un biholomorphisme n'altère pas le rang d'une fonction holomorphe on tire de cette égalité que le rang de $\pi_{i}^{\prime}$ est le même en tout point. De ce fait $\pi_{i}^{\prime}$ est régulière et $\mathcal{F}_{i}^{\prime}$ l'est aussi. Le même argument de translation montre que si les feuilletages $\mathcal{F}_{i}^{\prime}$ ne sont pas en position générale en un certain point, alors ils ne le sont nulle part et il existe de ce fait un champ de droites holomorphe $\Delta$ contenu en même temps dans le fibré tangent de $n$ des feuilletages de la famille $\mathcal{F}_{i}^{\prime}$. Ce champ est alors tangent à un feuilletage de dimension un inclus dans chacun de nos $n$ feuilletages, ce qui est absurde vu la construction. On conclut donc que les $\mathcal{F}_{i}^{\prime}$ sont bien en position générale.

LEMME 5.2. - Il existe un biholomorphisme $\psi$ qui envoie $z_{0}^{\prime}$ sur $z_{0}$ et $\mathcal{F}_{i}^{\prime}$ sur $\mathcal{F}_{i}$ pour tout $i \in\{0, \ldots, n\}$.

Preuve. - Il existe en $z_{0}^{\prime}$ des coordonnées locales holomorphes $\left(y_{1}, \ldots, y_{n}\right)$ dans lesquelles chaque $\mathcal{F}_{i}^{\prime}(1 \leq i \leq n)$ est défini par l'équation $d y_{i}=0$ de même indice, car les $\mathcal{F}_{i}^{\prime}$ sont $n$ feuilletages holomorphes réguliers et transverses au voisinage de $z_{0}^{\prime}$. Nous disons qu'un tel système de coordonnées est feuilleté (par rapport aux $\mathcal{F}_{i}^{\prime}$ d'indice $i>0$ ). Un système feuilleté topologique sera un système feuilleté dont les coordonnées sont seulement continues et non nécessairement holomorphes. On voit sans peine que si $\left(y_{1}, \ldots, y_{n}\right)$ et $\left(u_{1}, \ldots, u_{n}\right)$ sont deux systèmes feuilletés, le second est relié au premier par des formules de la forme $u_{i}=\psi_{i}\left(y_{i}\right)$ où les $\psi_{i}$ sont des fonctions continues (et holomorphes si les deux systèmes le sont). De ce fait, un $(n+1)$-ième feuilletage holomorphe $\mathcal{F}$ étant donné d'avance et en position générale par rapport aux $\mathcal{F}_{i}^{\prime}$, il y a une obstruction à ce qu'on puisse trouver une carte feuilletée où $\mathcal{F}$ soit défini par l'équation $d y_{1}+\cdots+d y_{n}=0$ : en effet si ce problème admet une solution $\left(u_{1}, \ldots, u_{n}\right)$, alors dans tout autre système feuilleté l'équation de $\mathcal{F}$ a la forme $d\left(\phi_{1}\left(y_{1}\right)+\right.$ $\left.\cdots+\phi_{n}\left(y_{n}\right)\right)=0$, ce qui n'est pas la forme la plus générale d'une équation de feuilletage. On dira qu'un feuilletage $\mathcal{F}$ pour lequel cette obstruction n'existe pas est holomorphiquement redressable, et qu'il est topologiquement redressable s'il en existe un redressement continu. Par construction, $\mathcal{F}_{0}$ est topologiquement redressable; ce qu'il faut montrer est que $\mathcal{F}_{0}$ est redressable. Mais on peut voir que l'obstruction à la redressabilité est topologique : en effet s'il existe un système feuilleté topologique redressant $\mathcal{F}$, alors l'équation de $\mathcal{F}$ dans tout système feuilleté holomorphe est de la forme $d\left(\phi_{1}\left(y_{1}\right)+\cdots+\phi_{n}\left(y_{n}\right)\right)=0$ et par transversalité, les fonctions $\phi_{i}$ sont toutes bijectives; en posant $u_{i}=\phi_{i}\left(y_{i}\right)$,

TOME $129-2001-\mathrm{N}^{\mathrm{O}} 2$ 
on obtient alors un redressement holomorphe de $\mathcal{F}$. Par conséquent $\mathcal{F}_{0}^{\prime}$ est redressable et le lemme est démontré.

Considérons la fonction $\psi \circ \phi$ : c'est une fonction continue qui fixe chacun des $\mathcal{F}_{i}$. Alors, cette transformation est feuilletée, et on peut donc écrire

$$
\psi \circ \phi\left(x_{1}, \ldots, x_{n}\right)=\left(\phi_{1}\left(x_{1}\right), \ldots, \phi_{n}\left(x_{n}\right)\right) .
$$

En outre, puisque l'image inverse de $\mathcal{F}_{0}$ par $\psi \circ \phi$ est le feuilletage d'équation $d\left(\phi_{1}\left(x_{1}\right)+\cdots+\phi_{n}\left(x_{n}\right)\right)=0$, mais est en même temps égal à $\mathcal{F}_{0}$, donné par $d x_{1}+\cdots+d x_{n}=0$, on voit qu'il existe une fonction $\phi_{0}$ telle que

$$
\left(x_{1}+\cdots+x_{n}=k\right) \Longrightarrow\left(\phi_{1}\left(x_{1}\right)+\cdots+\phi_{n}\left(x_{n}\right)=\phi_{0}(k)\right) \text {. }
$$

Prenant d'abord tous les $x_{i}$ nuls sauf un seul d'entre eux, on en tire $\phi_{1}=\cdots=$ $\phi_{n}=\phi$. Ensuite, prenant tous les $x_{i}$ nuls pour $i \geq 3$, on voit que $\phi\left(x_{1}\right)+\phi\left(x_{2}\right)=$ $\phi\left(x_{1}+x_{2}\right)$, donc la fonction continue $\phi$ est additive, ce qui implique qu'elle est $\mathbb{R}$-linéaire. On a donc finalement $\psi \circ \phi \in \mathrm{GL}(2 n, \mathbb{R})$. Mais par hypothèse, $\psi \circ \phi$ stabilise l'adhérence $\operatorname{GL}(n, \mathbb{C})$ de $\Gamma$ dans $\operatorname{GL}(2 n, \mathbb{R})$ donc aussi le centre $\mathbb{C}^{*}$ de ce groupe, et le sous-ensemble $\{ \pm i\}$ des éléments d'ordre 4 de ce centre. Par conséquent, $\psi \circ \phi(i z)= \pm i z$, ce qui veut bien dire que cette fonction est holomorphe ou antiholomorphe. Comme $\psi$ est elle-même holomorphe, le théorème est démontré.

\section{BIBLIOGRAPHIE}

[1] Anosov (D.V.), Arnold (V.I.) (eds.) - Dynamical systems I, E.M.S. 1 (Springer-Verlag).

[2] Belliart (M.), Liousse (I.), Loray (F.) - The generic rational differential equation $d w / d z=P_{n}(w, z) / Q_{n}(w, z)$ on $\mathbb{C P}^{2}$ carries no interesting transverse structure, to appear.

[3] Borel (A.) - Linear algebraic groups (2nd enlarged edition), Springer GTM 126.

[4] Cartan (É.) - Leçons sur la géométrie projective complexe, éditions Jacques Gabay.

[5] Elizarov (P.M.), Il'Yashenko (Y.S.), Scherbakov (A.A.), VoRONIN (S.M.) - Finitely generated groups of germs of one-dimensional conformal mappings, and invariants for complex singular points of analytic foliations of the complex plane, Adv. in Soviet Math., 14 (1993).

[6] GHys (E.) - Sur les groupes engendrés par des difféomorphismes proches de l'identité, Bol. Soc. Bras. Mat. (N.S.), 24 (1993), p. 137-178.

[7] Gromov (M.) - Rigid transformation groups, Hermann (1998). 
[8] IL'YASHenko (IU.S.) - The topology of phase portraits of analytic differential equations in the complex projective plane (Russian), Trudy Sem. Petrovsk, 4 (1978), p. 83-136.

[9] LAmy (S.) - Automorphismes polynomiaux du plan complexe : étude algébrique et dynamique, Thèse (Toulouse, janvier 2000).

[10] Loray (F.), Rebelo (J.) - Stably chaotic rational vector fields on $\mathbb{C P}^{n}$, à paraître.

[11] NAKAi (I.) - Separatrices for non solvable dynamics on $\mathbb{C}, 0$, Ann. Inst. Fourier, 44-2 (1994), p. 569-599.

[12] Shcherbakov (A.) - On the density of an orbit of a pseudogroup of conformal mappings and a generalisation of the Hudai-Veronov theorem (Russian), Vestnik Movskovskogo Universiteta Mathematica, 31-4 (1982), p. 10-15. 